\title{
Precocious Downregulation of Krüppel-Homolog 1 in the Migratory Locust, Locusta migratoria, Gives Rise to An Adultoid Phenotype with Accelerated Ovarian Development but Disturbed Mating and Oviposition
}

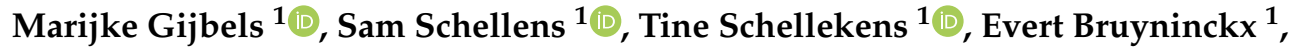 \\ Elisabeth Marchal 1,2,*(D) and Jozef Vanden Broeck ${ }^{1, *(D)}$ \\ 1 Research Group of Molecular Developmental Physiology and Signal Transduction, KU Leuven, Zoological \\ Institute, Naamsestraat 59 box 2465, 3000 Leuven, Belgium; Marijke.Gijbels@kuleuven.be (M.G.); \\ Sam.Schellens@kuleuven.be (S.S.); Tine_schellekens@hotmail.com (T.S.); \\ Evert.Bruyninckx@kuleuven.be (E.B.) \\ 2 Life Science Technologies, Imec, Kapeldreef 75, B- 3001 Leuven, Belgium \\ * Correspondence: Elisabeth.Marchal@imec.be (E.M.); Jozef.VandenBroeck@kuleuven.be (J.V.B.)
}

Received: 4 August 2020; Accepted: 20 August 2020; Published: 22 August 2020

\begin{abstract}
Krüppel-homolog 1 (Kr-h1) is a zinc finger transcription factor maintaining the status quo in immature insect stages and promoting reproduction in adult insects through the transduction of the Juvenile Hormone (JH) signal. Knockdown studies have shown that precocious silencing of $K r-h 1$ in the immature stages results in the premature development of adult features. However, the molecular characteristics and reproductive potential of these premature adult insect stages are still poorly understood. Here we report on an adult-like or 'adultoid' phenotype of the migratory locust, Locusta migratoria, obtained after a premature metamorphosis induced by the silencing of $L m K r-h 1$ in the penultimate instar. The freshly molted adultoid shows precocious development of adult features, corresponding with increased transcript levels of the adult specifier gene LmE93. Furthermore, accelerated ovarian maturation and vitellogenesis were observed in female adultoids, coinciding with elevated expression of $L m C Y P 15 A 1$ in corpora allata (CA) and $L m K r-h 1$ and vitellogenin genes $(L m V g$ ) in fat body, whereas LmE93 and Methoprene-tolerant ( $L m M e t)$ transcript levels decreased in fat body. In adultoid ovaries, expression of the Halloween genes, Spook (LmSpo) and Phantom ( $\mathrm{LmPhm}$ ), was elevated as well. In addition, the processes of mating and oviposition were severely disturbed in these females. L. migratoria is a well-known, swarm-forming pest insect that can destroy crops and harvests in some of the world's poorest countries. As such, a better understanding of factors that are capable of significantly reducing the reproductive potential of this pest may be of crucial importance for the development of novel locust control strategies.
\end{abstract}

Keywords: ecdysteroid; Hemimetabola; insect; juvenile hormone; metamorphosis; reproduction

\section{Introduction}

The transition from immature insect stages to fully reproductive adults is dependent on two hormones, i.e., Juvenile Hormone (JH) and ecdysteroids. Whereas periodic pulses of ecdysteroids trigger the molting process in juvenile insects, the absence or presence of $\mathrm{JH}$ will determine the nature of the molt [1]. JH is a sesquiterpenoid hormone synthesized in the corpora allata (CA), which are part of the retrocerebral complex of insects. In addition to their role in immature insect stages, both ecdysteroids and $\mathrm{JH}$ are endocrine regulators of reproduction in adult insects. Ecdysteroids induce their effects by binding to a heterodimeric complex of two nuclear receptors, the ecdysone 
receptor (EcR) and the retinoid-X-receptor/ultraspiracle (RXR/USP), as reviewed by Hill et al. (2013) [2]. The JH signaling pathway was only recently described with the in vitro and in vivo characterization of a genuine $\mathrm{JH}$ receptor, Methoprene-tolerant (Met). Met is a transcription factor belonging to the basic helix-loop-helix (bHLH)/Per-Arnt-Sim (PAS) family [3,4]. The binding of JH stimulates Met to form a complex with other bHLH-PAS proteins such as Taiman (Tai), a steroid receptor co-activator. The JH-Met-Tai complex then induces the expression of JH response genes such as Krüppel-homolog 1 $(K r-h 1)$, the main effector of the anti-metamorphic action of JH $[5,6]$. In a variety of insect species, RNA interference (RNAi) studies have demonstrated the importance of Met and its downstream transcription factor Kr-h1 in the anti-metamorphic effects of JH. Knockdown of either Met or Kr-h1 in larvae of the red flour beetle, Tribolium castaneum, induced a precocious metamorphosis to pupae [7-9]. Reduced Met or $K r-h 1$ transcript levels in nymphs of the German cockroach, Blattella germanica, the kissing-bug, Rhodnius prolixus, the cricket, Gryllus bimaculatus, the brown planthopper, Nilaparvata lugens, the common bed bug, Cimex lectularius, and the fire bug, Pyrrhocoris apterus, resulted in precocious development of adult features [10-17]. Although the occurrence of a precocious metamorphosis resulting in adultoid insects is well known, its impact on reproductive physiology is only poorly understood. Nowadays, only the morphology of reproductive organs has been described in adultoids of a few species. In adultoids of the bed bug, C. lectularius, induced by RNAi-mediated knockdown of $K r-h 1$, precocious development of ovaries and oviducts was observed [17]. In addition, external genitals were found in $d s K r-h 1$-injected N. lugens and P. apterus $[10,16,18]$, while an aberrant ovipositor morphology was described in adultoid crickets of the species G. bimaculatus [15].

While Kr-h1 mediates the status quo action of JH, the ecdysteroid early response gene, E93, is a key determinant promoting adult morphogenesis in both holo- and hemimetabolan insects. In nymphs of B. germanica, G. bimaculatus, C. lectularius and N. lugens, E93 depletion prevented the nymphal-to-adult transition, giving rise to supernumerary nymphal instars [15-17,19]. In the holometabolan insect T. castaneum, E93 depletion in larvae prevented the larval-to-pupal or pupal-to-adult transition, resulting in the formation of a supernumerary second pupa or inducing reiteration of larval development depending on the time of double-stranded (ds)RNA injection $[9,19]$. These studies have established the inhibitory effect of $\mathrm{Kr}-\mathrm{h} 1$ on E93 expression and vice versa $[9,19,20]$. This ancestral regulatory mechanism by which JH inhibits metamorphosis is now defined as the MEKRE93 axis. During nymphal-to-nymphal transitions, high JH titers induce $K r$ - $h 1$ expression (via Met), which in turn represses the expression of E93. In the final juvenile stage, when JH titers drop, $K r$ - $h 1$ expression is interrupted, resulting in a strong induction of E93 through ecdysone signaling, which triggers adult development (as reviewed by Belles, 2019) [21].

Moreover, in adult insects, JH transduces its signal via Met and Kr-h1. Multiple studies have confirmed the involvement of $\mathrm{JH}$ in vitellogenin synthesis in the fat body, lipid accumulation in the primary oocyte, regulation of mating and sex pheromone production in several insects [22-36]. Moreover, an effect on the number of eggs deposited could be attributed to JH as well, since a reduced number of eggs laid was observed upon silencing Met or $K r-h 1$ in the brown planthopper, N. lugens [22]. Furthermore, in locusts, such as L. migratoria, RNAi-studies have shown the importance of Met and $K r-h 1$ in female reproductive physiology. A knockdown of these genes caused lowered vitellogenin $(V g)$ expression in the fat body, reduced lipid accumulation in the oocytes and inhibition of patency in the follicular epithelium, thereby preventing oocyte growth [6,37]. A recent study in the desert locust, Schistocerca gregaria, confirmed these findings, since a knockdown of Met in female adult locusts resulted in a failure to initiate $V g$ expression in the fat body, as well as basal oocyte growth in the ovaries. Moreover, these dsMet-treated locusts showed delayed mating behavior and were unable to oviposit [38]. In female locusts, $\mathrm{JH}$ is known to stimulate $\mathrm{Vg}$ production by inducing $\mathrm{Vg}$ gene transcription in the fat body [39]. Via binding of $\mathrm{JH}$ to membrane receptor sites in the ovarian follicular epithelium, a process called patency, the shrinkage of cells, is initiated [40-43]. As a result, Vgs, which are mainly produced by the fat body and then released into the hemolymph, can be taken up by the developing oocyte. Moreover, an oviduct derived Patency Inducing Factor was recently discovered. 
This factor, clearly boosted by JH, is involved in the initiation of patency in the terminal follicle via the pedicel [44]. Apart from $\mathrm{JH}$, ecdysteroids also play an important role in the reproductive physiology of female locusts. Produced in the ovarian follicular cell layer, they appear to induce meiotic re-initiation in the oocyte [45] and they are incorporated as conjugates into the eggs where they act as a source of ecdysteroids during embryogenesis [46]. Moreover, they can bind to vitellins, thereby preventing their leakage into the hemolymph [46-48]. Furthermore, in the desert locust S. gregaria, the ecdysteroid receptor complex is playing a crucial role in chorion formation [49].

Locust swarms are well known for their ability to destroy the agricultural production in some of the world's poorest countries [50-52]. A major upsurge of L. migratoria in Madagascar reached biblical plague proportions in 2013 [53]. This plague endangered the food security of 13 million people and 2.3 million hectares of land had to be treated with insecticides in three consecutive control campaigns that lasted until July 2016. Moreover, several other locust species can form devastating swarms on different continents. Global warming together with poor monitoring due to political issues very recently led to a huge plague of desert locusts (S. gregaria) that are currently swarming through large parts of East Africa, the Middle East and the Indian subcontinent, threatening food supply and livelihoods of at least 20 million people [54]. Furthermore, in Sardinia, the recently occurring swarms of another species, the Moroccan locust (Dociostaurus maroccanus), have been described as the worst invasion in 60 years [55,56]. Unfortunately, the control of locust plagues mainly relies on the use of classic broad-spectrum insecticidal nerve toxins, which can result in a negative impact on the environment. Therefore, in the fight against locusts there is an urgent need for greener, more sustainable pest management strategies. Components of pathways regulating important insect processes, such as metamorphosis and reproduction, as investigated in the current study, would make for very attractive molecular targets of novel insect control agents.

Our study describes a premature adult-like (adultoid) L. migratoria phenotype, the result of a reprogrammed development caused by the precocious RNAi-mediated silencing of $L m K r-h 1$ during the fourth (and normally penultimate) nymphal stage. Adultoid locusts are characterized by an adult color scheme and the presence of wings, although not fully extended, while maintaining the physical size of a fifth nymphal locust. Whereas several studies in different insect species already described the remarkable morphological consequences of such a knockdown, here we primarily focus on the reproductive properties of these adultoids and compare these with normal adult females. In this context, we analyze and compare the expression of genes contributing to the MEKRE93 axis, vitellogenesis and hormone biosynthetic pathways during the first gonadotrophic cycle of both adult and adultoid females. Moreover, effects on mating, oviposition, fecundity and fertility are monitored as well.

\section{Results}

\subsection{Temporal Transcript Profiles of MEKRE93 and JH Biosynthesis Pathway Components}

In female locusts (double-stranded green fluorescent protein (dsGFP)-injected control), we investigated the temporal profiles of the MEKRE93 pathway transcripts LmKr-h1, LmE93 and LmMet, as well as these of LmJHAMT and LmCYP15A1, the enzymes catalyzing the two final steps in JH biosynthesis, by qRT-PCR analyses at different time points from day 4 of the fourth nymphal (N4 D4) until day 12 of the adult (Ad D12) stage (Figure S1). Relative $L m K r$ - $h 1$ transcript levels were studied in the fat body, ovaries and CA. In the fat body, $L m K r$ - $h 1$ was highly expressed at the end of the fourth nymphal stage (N4 D4). After molting to fifth nymphal locusts (N5 D0), LmKr-h1 levels were significantly lower and stayed low at the following time point until initiating the adult molt (N5 D6 and Ad D0) when significant increases were observed (Figure S1A). A gradual increase in LmKr-h1 expression was observed in the ovaries of adult females (Figure S1C), whereas in the CA (Figure S1B), reduced $L m K r$ - $h 1$ levels were detected on the days of molting (N5 D0, Ad D0) and when the ovaries had entered the vitellogenic stage (Ad D12). At the two earliest time points, situated before and after the 
N4-N5 molt (N4 D4 and N5 D0), LmE93 levels were low in both fat body and CA, but rose significantly during the final nymphal stage (N5 D3) (Figure S1D,E). In fat body, LmE93 expression remained high at the different time points in the adult stage, while in the CA of the adult stage they gradually decreased towards day 12 after the final molt (Ad D12). In addition, LmMet expression levels in fat body were high on day 4 of the fourth nymphal stage (N4 D4) followed by a drop towards the fifth nymphal stage (N5 D0), after which they remained relatively constant at the following time points in this nymphal stage. In fat body of adult females, significantly lower LmMet levels were detected on days 4 and 12 versus higher ones on days 0 and 8 (Figure S1G). LmMet expression levels measured in the CA showed a decreasing trend throughout the different time points of the final instar and adult stage. For checking the expression of two JH biosynthetic enzymes, the site of JH biosynthesis, i.e., the CA, was selected. Both LmJHAMT and LmCYP15A1 showed a significant increase in their expression after the transition from fifth nymphal to adult locusts (Ad D0), and LmJHAMT further increased on day four of the adult stage (Ad D4). The relative expression levels of both genes remained high at the time points observed during adult life (Figure S1F,I).

\subsection{Knockdown of LmKr-h1 Results in Precocious Development of External Adult Features}

An initial RNAi pilot study was performed to eliminate the possibility of off-target effects and to verify the reproducibility of the phenotypic effects. Therefore, two distinct dsRNA constructs were designed (dsLmKr-h1 and $d s L m K r$ - $h 1 \_2$; Figure S2). When tested within the same experimental setup, similar phenotypic effects were observed with both constructs. Next, the $d s L m K r-h 1$ construct was utilized in all further experiments.

The RNAi treatment, based on injections of $d s L m K r-h 1$ during the fourth nymphal stage (on D0 and D3), significantly affected development of L. migratoria (Figure 1). In total, 93\% of the experimental locusts did not molt into the fifth nymphal stage, while $100 \%$ of the $d s G F P$-injected (control) locusts successfully completed this nymphal-to-nymphal molt (Figure 1A-C). Instead of molting into fifth nymphal locusts, most of the $d s L m K r$ - $h 1$-injected (experimental) animals molted into an adultoid phenotype, showing precocious development of adult features. These adultoids already had an adult color scheme as well as the presence of wings, although not fully extended and non-functional, while maintaining the physical body size of a fifth nymphal locust (Figure 1A). The animals that molted to the adultoid phenotype had remained in the fourth nymphal stage for a longer time than the dsGFP-injected (control) locusts that molted to the fifth nymphal stage: their N4 stage lasted for 6-8 days compared to 4-6 days, respectively (Figure 1C). Moreover, the adultoid insects did not undergo any further molts (Figure 1D). Apart from the adultoid phenotype, 29\% of the $d s L m K r$-h1-injected (experimental) animals molted into an intermediate phenotype (Figure 1B), still having a nymphal color scheme, but with more developed wings (Figure 1A). These animals had remained in the fourth nymphal stage for 5 to 7 days before molting to their intermediate phenotype (Figure 1C). Unlike the adultoid phenotype, $20 \%$ of the intermediate phenotype locusts were still successfully completing the adult molt, while $100 \%$ of the control $(d s G F P)$ and experimental (dsLmKr-h1) fifth nymphal locusts successfully completed the adult molt (Figure 1D). 
A
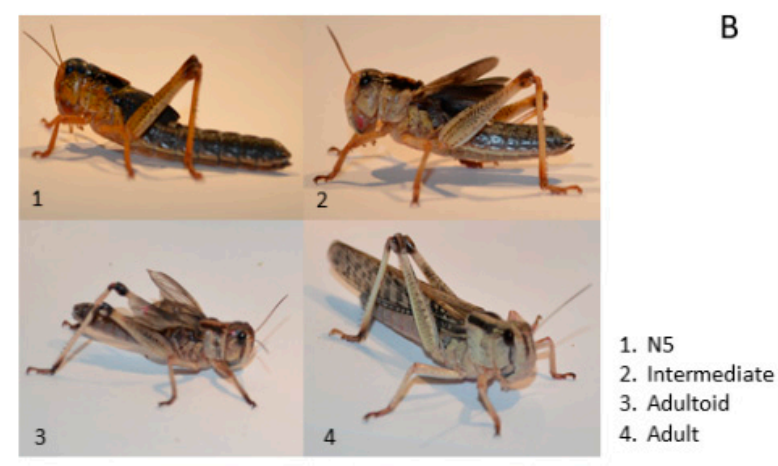

7 - 7 N5 $\square 29 \%$ Interm. $\square 64 \%$ Ao

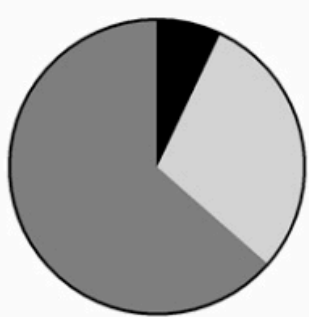

C
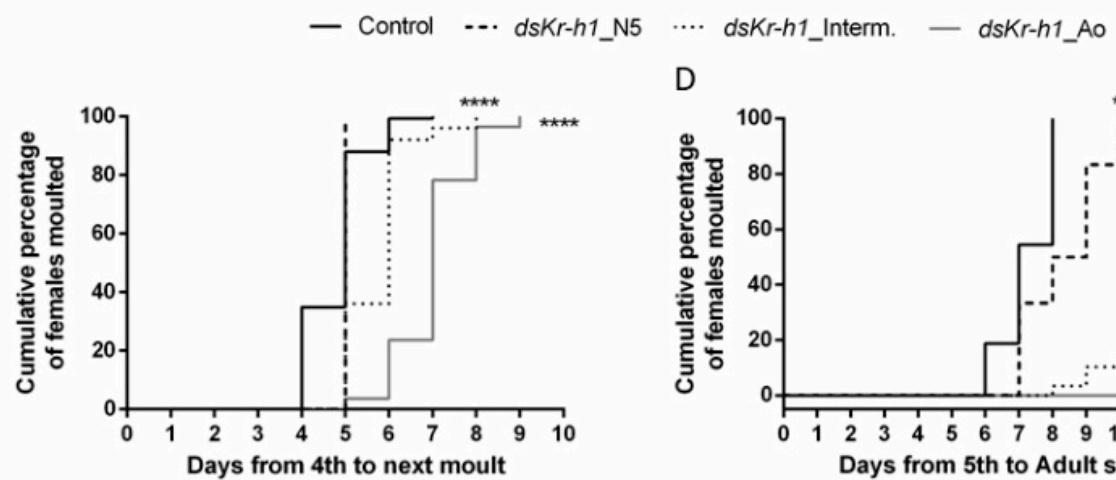

D

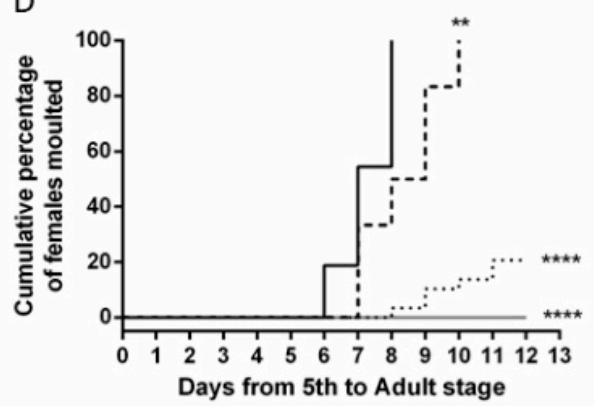

Figure 1. Phenotypes of $d s L m K r-h 1$-injected female locusts and the timing of their ecdysis. The timing of ecdysis of $148 d s G F P$ (control) and $83 d s L m K r$ - $h 1$-injected female locusts was observed starting from freshly molted fourth nymphal locusts. (A) Observed phenotypes of $d s G F P$ (control) and/or $d s L m K r$ - $h 1$-injected locusts obtained after molting: (1) fifth nymphal stage (N5), (2) intermediate (Interm.), (3) adultoid (Ao), (4) adult (Ad). (B) A pie chart representing the percentages of phenotypes (N5, Interm, Ao) observed after molting of the $d s L m K r$-h1-injected fourth nymphal (N4) locusts. (C) The cumulative percentage of females that molted from the fourth nymphal to the fifth nymphal (control and $\left.d s K r-h 1 \_N 5\right)$ or to a precocious adult-like stage, classified as an intermediate (dsKr-h1_Interm.) or an adultoid phenotype ( $d s K r-h 1 \_$Ao). (D) The cumulative percentage of females that molted from N5, Interm. or Ao to the adult stage. Statistically significant differences $(p)$ between the conditions were found via a log-rank (Mantel-Cox) test and are indicated by asterisks $\left({ }^{* *} p<0.01\right.$; $\left.{ }^{* * *} p<0.0001\right)$.

2.3. Transcript Levels of MEKRE93 and JH Biosynthesis Pathway Components in dsRNA-Injected Fourth Nymphal and Freshly Molted Fifth Nymphal, Adultoid and Adult Female Locusts

Transcript levels of different components of the MEKRE93 pathway, as well as the enzymes catalyzing the two final steps of $\mathrm{JH}$ biosynthesis, were determined in different tissues on two developmental time points, i.e., day 4 of the fourth nymphal stage (N4 D4) and freshly molted fifth nymphal (N5 D0), adult (Ad D0) or adultoid (Ao D0) locusts (Figure S3). A significant downregulation of $L m K r-h 1$ transcript levels was measured in the fat body (44\%) of fourth nymphal $d s L m K r-h 1$-injected locusts, when compared with $d s G F P$-injected (control) locusts of the same age (N4 D4; Figure S3A). While in the ovaries (Figure S3C) no significant change in the transcript level was observed at this time point, a significant increase was noticed in the CA (Figure S3B). Interestingly, on the day of molting, $L m K r-h 1$ relative transcript levels were significantly higher in fat body and ovaries of adultoid locusts than fifth nymphal ones (Figure S3A,C), while no significant differences were observed in the CA (Figure S3B).

In comparison with $d s G F P$-injected (control) locusts, the LmE93 mRNA levels were significantly higher in both fat body and CA of $d s L m K r-h 1$-injected locusts on day 4 of the fourth instar (N4 D4). On the day of molting, the $L m E 93$ transcript levels in both tissues were significantly higher in adultoid $(d s L m K r-h 1)$ than in fifth nymphal locusts (dsGFP control), while no significant differences 
were observed between adultoid and adult locusts (Figure S3D,E). For LmMet mRNA levels, on the other hand, no significant changes were noticed with the single exception of a significantly lower level in the fat body of freshly molted fifth nymphal (dsGFP control) locusts (Figure S3G,H). When checking the expression levels of two JH biosynthetic enzymes, LmJHAMT and LmCYP15A1 in the CA, no significant differences were observed between $d s L m K r-h 1$ and $d s G F P$-injected locusts on the time points that were analyzed (Figure S3F,I).

\subsection{The Adultoid Phenotype Shows an Accelerated Ovarian Maturation}

To investigate the effect of the RNAi-mediated knockdown of $L m K r-h 1$ in fourth nymphal locusts on the later female reproductive physiology, adult (dsGFP control) and adultoid (dsLmKr-h1) locusts were dissected every four days (Figure 2A). Adultoid female locusts were in full vitellogenesis eight days after their precociously induced final molt (Ao D8). In comparison, eight days after their final molt (Ad D8) the adult females included in the same experiment had an average oocyte length of only $1.39 \mathrm{~mm}$. Moreover, considering that a fifth nymphal stage preceded their adult molt, as illustrated in Figure 2A, the locusts of the control group $(d s G F P)$ were, in total, at least four days older than the adultoid (dsLmKr-h1) females. Adultoid females did not only develop faster but their ovaries also matured faster, as shown by the growth of their basal oocytes over time (Figure 2B). On day 4 of the adult stage, the average basal oocyte length measured $0.62 \mathrm{~mm}$, while in adultoids this was $0.53 \mathrm{~mm}$. Significantly accelerated growth was observed on day 8 of the adultoid phenotype with the average oocyte length of $3.88 \mathrm{~mm}$, more than double the size of the basal oocytes in day 8 adults measuring $1.39 \mathrm{~mm}$. A significant length difference was also observed between the basal oocytes in adultoids and adults on day 12 after the final molt. Ovaries were further dissected, cleaned by removing the attached fat body and imaged (Figure $2 \mathrm{C}-\mathrm{E}^{\prime}$ and $\mathrm{M}-\mathrm{O}$ ). Some adultoid females were already in their second gonadotrophic cycle, characterized by orange lesions at the base of the new basal oocyte. In addition to these orange lesions, multiple resorbing oocytes were observed (Figure 2D-E'). Upon dissection of the adultoid females, it became clear that, while most of these animals were unable to deposit their eggs, fully grown oocytes were filling the oviducts (Figure 2E). Transverse sections of terminal oocytes were examined. The sections clearly confirmed the presence of yolk (blue matrix) and lipid droplets (grey spheres) from as early as eight days after the final molt to adultoid (Figure 2F-H), whereas in the oocytes of adult (control) locusts, the presence of yolk and lipid droplets was only observed at day 12 (Figure 2K,L). In the resorbing oocytes of adultoids, a loss of yolk was observed (Figure 2I,I'). The oocytes found in the oviducts of adultoid locusts contained yolk and lipid droplets and were clearly surrounded by a chorion (Figure 2J). 
A
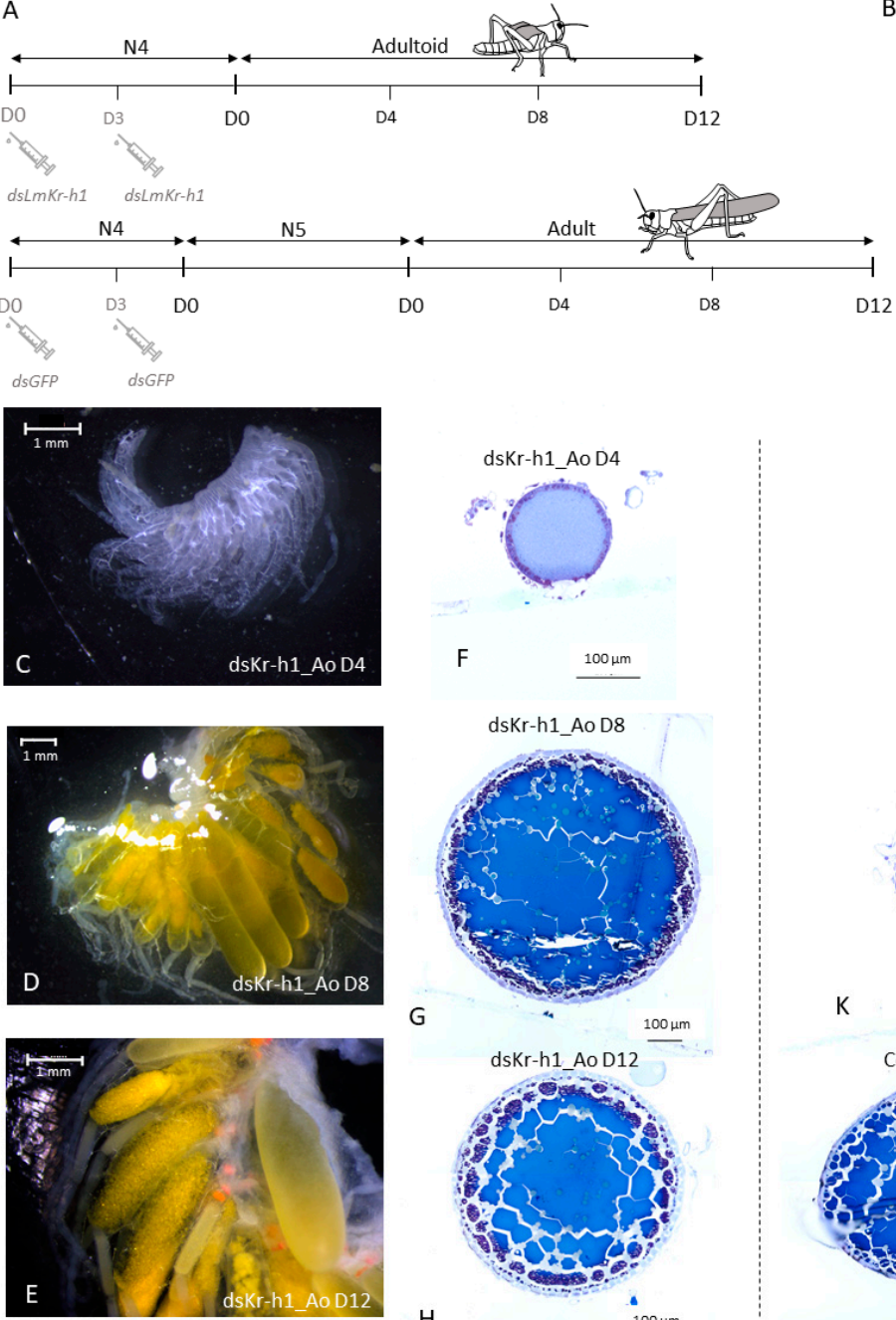

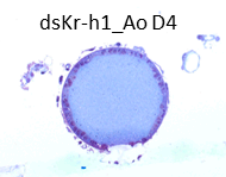

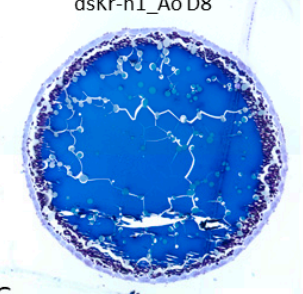

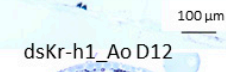

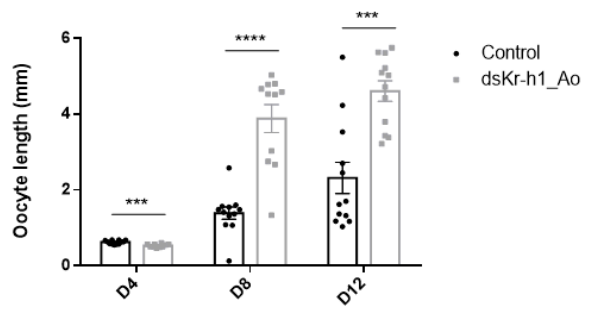
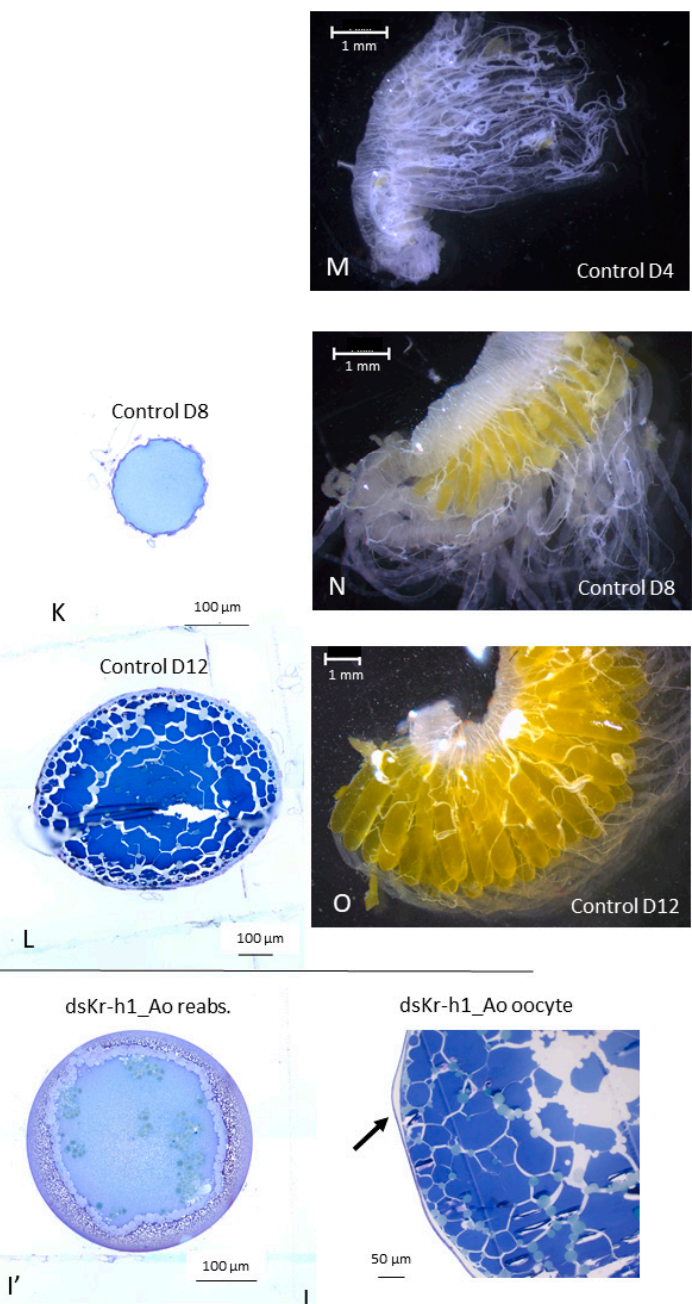

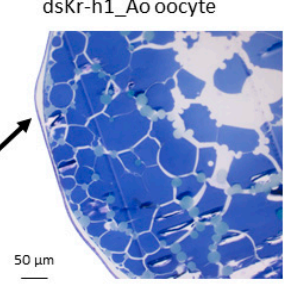

Figure 2. Ovarian maturation in adult and adultoid migratory locusts (L. migratoria). (A) Time scheme showing the different time points of dsRNA injection together with the duration of each developmental stage and the different time points chosen to dissect control adult (dsGFP) and adultoid (dsLmKr-h1) female locusts. (B) The oocyte length ( $\mathrm{mm}$ ) of adult (control: black bars) and adultoid (dsKr-h1_Ao: grey bars) female locusts was measured on day 4 (D4), day 8 (D8) and day 12 (D12) after the final molt. Each point represents the mean length of 3 randomly chosen basal oocytes (mean \pm S.E.M). Statistically significant differences $(p)$ were found via a nonparametric Mann-Whitney test and are indicated by asterisks ( $\left.{ }^{* * *} p<0.001 ;{ }^{* * * *} p<0.0001\right)$. (C-E' and $\left.\mathbf{M}-\mathbf{O}\right)$ Ovaries of 4-day-old (C), 8-day-old (D) and 12-day-old (E,E') adultoid (dsKr-h1_Ao) locusts compared to 4-day-old (M), 8-day-old (N) and 12-day-old (O) adult locusts (control). Scale bars: $1 \mathrm{~mm}$. (F-L) Histological sections of basal oocytes from 4-day-old (F), 8-day-old (G) and 12-day-old (H) adultoid (dsKr-h1_Ao) locusts compared to 8-day-old (K) and 12-day-old (L) adult locusts (control). Scale bar: $100 \mu \mathrm{m}$. (I-J) Histological sections of reabsorbing oocytes $\left(\mathbf{I}, \mathbf{I}^{\prime}\right)$ and an oocyte (J) found in the oviduct of an adultoid locust (dsKr-h1_Ao), with arrow (black) indicating the chorion. Scale bars: $100 \mu \mathrm{m}\left(\mathbf{I}, \mathbf{I}^{\prime}\right)$ and $50 \mu \mathrm{m}(\mathbf{J})$. 
Since yolk accumulation was observed relatively early in oocytes of adultoid ( $d s L m K r-h 1)$ females, $L m V g$ mRNA levels were analyzed in fat body samples during the first gonadotrophic cycle and compared to normal (dsGFP control) adult females (Figure 3). In the fat bodies of adultoid locusts, significantly higher $V g$ mRNA levels were observed for both $L m V g 1$ (Figure 3A) and $L m V g 2$ (Figure 3B) on days 8 and 12 after the final molt. When checking the temporal profiles of $L m V g$ mRNA transcripts in fat body samples of adult and adultoid female locusts during their first gonadotrophic cycle (Figure S4), significantly higher $L m V g$ mRNA levels were observed for both $L m V g 1$ (Figure S4A,C) and $L m V g 2$ (Figure S4B,D) on day 8 compared to day 4. Unlike adultoid females, $L m V g$ mRNA levels in control adult females were still significantly increasing towards day 12 (Figure S4A,B).

- Control adult $\square$ dsLmKr-h1 adultoid

A

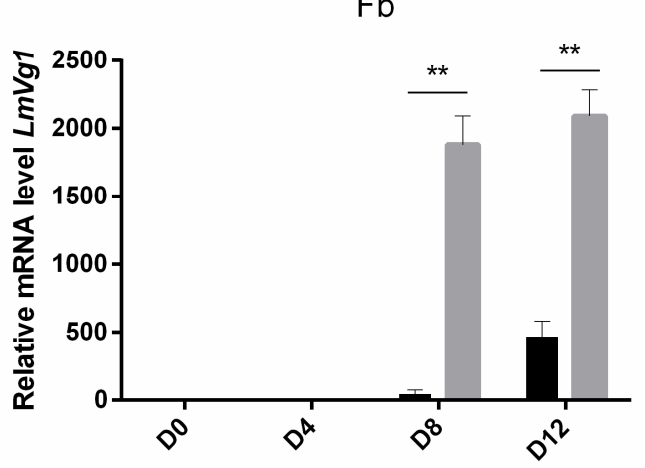

B

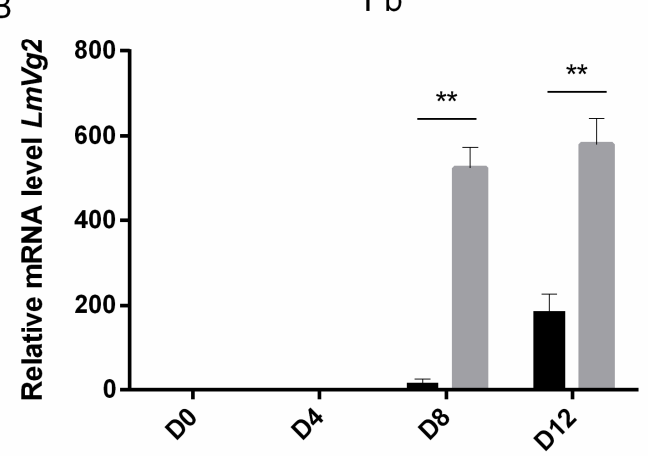

Figure 3. Relative $L m V g 1$ and $L m V g 2$ transcript levels in adult and adultoid females. Relative transcript levels of $L m V g 1$ (A) and $L m V g 2$ (B) in the fat body of freshly molted (D0), 4-day-old (D4), 8-day-old (D8) and 12-day-old (D12) adultoid (dsLmKr-h1) and adult (dsGFP: control) female locusts. The data represent mean \pm S.E.M. of four independent pools of three animals, run in duplicate and normalized to rps13 and CG13220 transcript levels. Statistically significant differences between the measurements were found via a $t$-test on log-transformed data (with or without two-sided Welch's correction) and are indicated by asterisks $\left.{ }^{* *} p<0.01\right)$.

\subsection{Transcript Levels of MEKRE93 and JH Biosynthesis Pathway Components in Adultoid Females}

The temporal expression profiles of genes encoding MEKRE93 and JH biosynthesis pathway components were monitored in the fat body, in the CA and in the ovaries of adultoid (dsLmKr-h1) female locusts (Figure S5). Figure 4 compares the expression levels at different time points after the final molt between adultoid ( $d s L m K r-h 1)$ and adult (dsGFP control) female locusts. In the fat body of adultoid female locusts, $L m K r$ - $h 1$ transcript levels significantly increased at day 8 (Figure S5A). At this time point a very pronounced increase was also observed in the ovaries (Figure S5C), whereas in the CA an increasing trend was observed from D4 to D12 (Figure S5B). When compared to adult females, the relative $L m K r-h 1 \mathrm{mRNA}$ levels of adultoid female locusts were significantly higher at different time points after the final molt: on days 4 and 12 in the fat body and day 12 in the CA (Figure $4 A, B$ ). In the fat body of adultoids, LmE93 and LmMet mRNA levels were significantly higher on the day of molting (Ao D0) and then decreased on the following time point (Ao D4) (Figure S5D,G). Furthermore, when compared to days 0 and 4, a very pronounced decrease in the LmE93 level was observed in the fat body of adultoids on day 8 (Ao D8) (Figure S5D). In the fat body of adult female locusts, a clear decreasing trend in the LmE93 and LmMet expression profiles was not observed (Figure S1D,G). Therefore, in comparison to the adults, the adultoids had significantly lower LmE93 and LmMet transcript levels in their fat body on day 8 after the final molt (Figure 4C,E). On the other hand, when compared to adult females, the adultoids had significantly higher LmE93 transcript levels in the CA at some time points (D4 and D12), while for LmMet no significant differences were observed between adults and adultoids in these JH producing endocrine organs (Figure 4D,F). In a Pearson correlation calculation, the temporal profiles of the relative mRNA levels of LmE93 and LmMet in the CA of control (dsGFP) 
adult females correlated well with these of adultoid (dsLmKr-h1) females (Figure 4D,F). For the two JH biosynthetic enzymes, LmJHAMT and LmCYP15A1, relative transcript levels tended to be higher in sexually maturing adultoid females (Figure S5F,I). When compared to adults, the adultoid females had significantly higher $L m C Y P 15 A 1$ levels in their CA on day 8 after the final molt (Figure $4 \mathrm{H}$ ).

- Control Adult

dsLmKr-h1 Adultoid

A

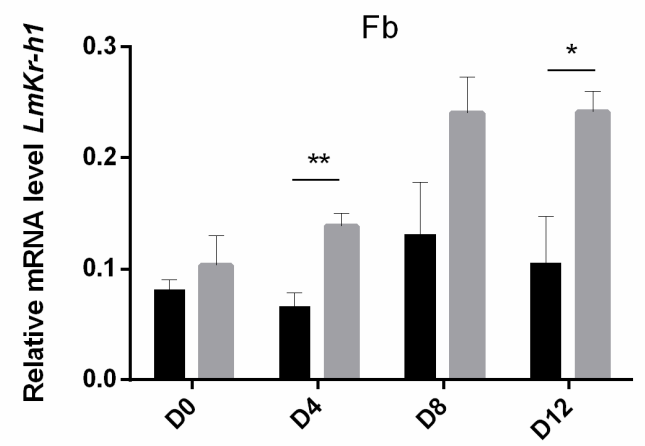

C

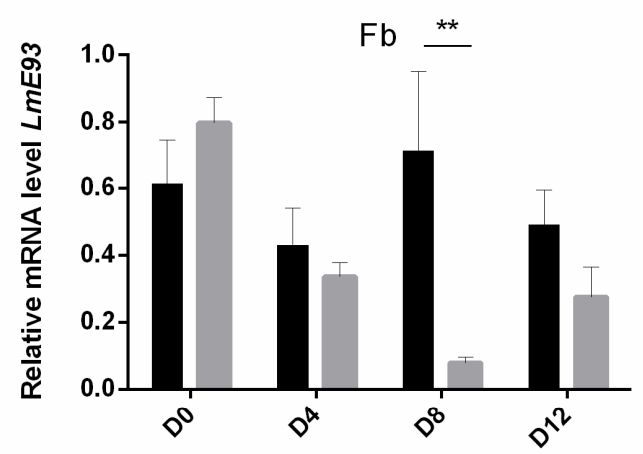

E

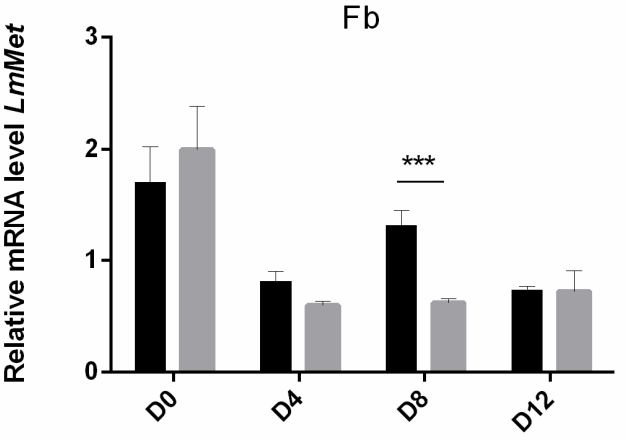

G

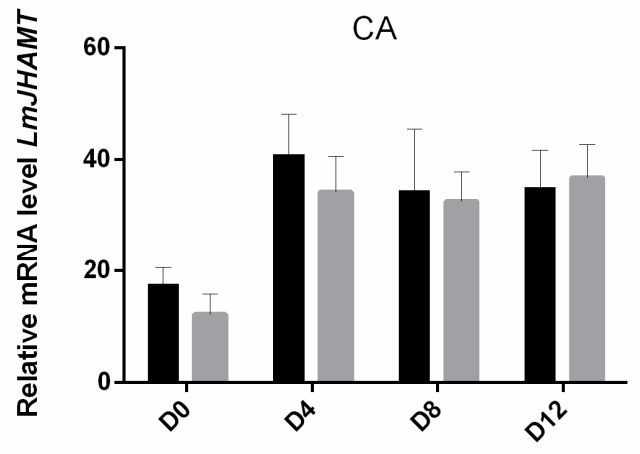

$\mathrm{B}$

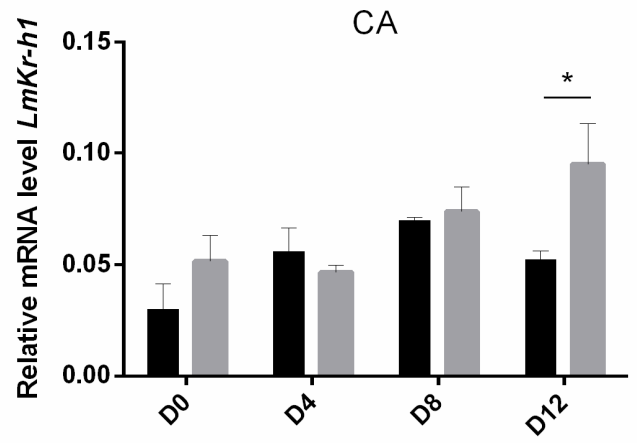

$\mathrm{D}$

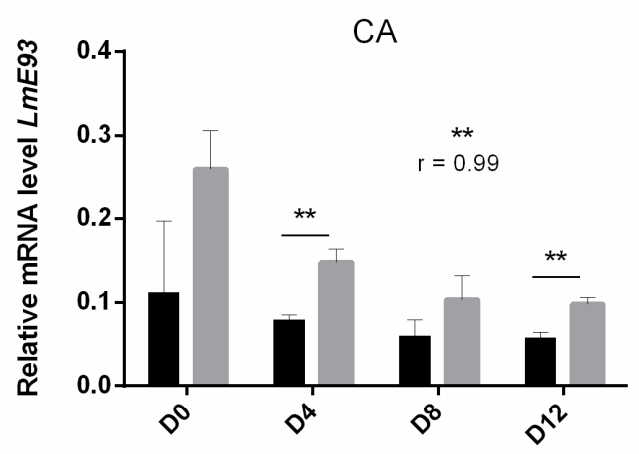

$\mathrm{F}$

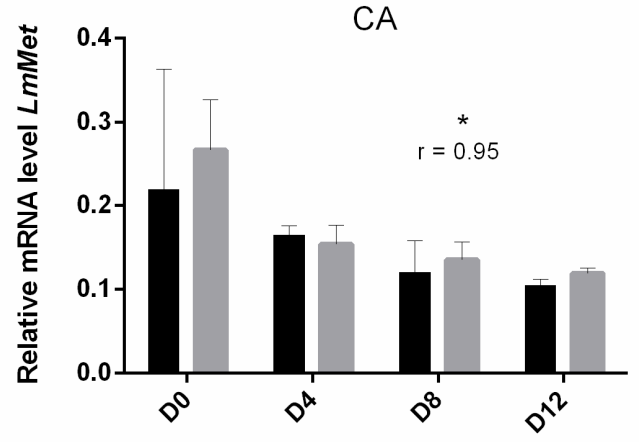

$\mathrm{H}$

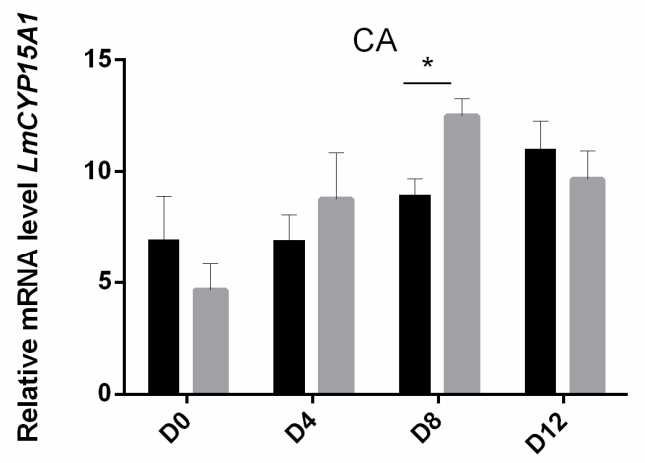

Figure 4. Relative transcript levels of MEKRE93 and JH biosynthesis pathway components in adult and adultoid L. migratoria females. Relative $\operatorname{LmKr}-h 1(\mathbf{A}+\mathbf{B}), \operatorname{LmES3}(\mathbf{C}+\mathbf{D})$ and $L m M e t(\mathbf{E}+\mathbf{F})$ transcript 
levels were measured in the fat body $(\mathbf{A}+\mathbf{C}+\mathbf{D})$ and the $\mathrm{CA}(\mathbf{B}+\mathbf{D}+\mathbf{F})$ of freshly molted (D0), 4-day-old (D4), 8-day-old (D8) and 12-day-old (D12) adultoid (dsLmKr-h1) and adult (dsGFP: control) female locusts. Relative $\operatorname{LmJHAMT}(\mathbf{G})$ and $\operatorname{LmCYP15A1}(\mathbf{H})$ transcript levels were measured in the $\mathrm{CA}$ as well. The data represent mean \pm S.E.M. of four independent pools of three animals, run in duplicate and normalized to rps13 + CG13220 and rp49 + rps13 transcript levels for fat body and CA samples, respectively. Statistically significant differences between the measurements were found via a $t$-test on log-transformed data (with or without two-sided Welch's correction) and are indicated by (an) asterisk(s) $\left.{ }^{*} p<0.05 ;{ }^{* *} p<0.01 ;{ }^{* *} p<0.001\right)$. The correlation coefficient (r) between the transcript profiles of adult and adultoid locusts was obtained by a Pearson correlation calculation. This coefficient is indicated above the figure panels where a significant correlation was observed [ $p$-value is indicated by (an) asterisk(s) (* $\left.p<0.05 ;{ }^{* *} p<0.01\right)$ ].

\subsection{Transcript Levels of Halloween Genes and Ecdysone Receptor Complex in Adultoids And Adults}

Ovaries were collected every four days starting from freshly molted (D0) adultoid and adult locusts until day 12 (D12) after the final molt. Figure 5 compares the ovarian expression levels of LmKr-h1, the Halloween genes, Spook (LmSpo), Phantom (LmPhm), Disembodied (LmDib), Shadow (LmSad) and Shade (LmShd), which code for cytochrome P450 enzymes involved in ecdysteroid biosynthesis, as well as of the $L m E c R$ and $L m R X R$ genes, which encode the components of the ecdysone receptor complex, in adultoid ( $d s L m K r-h 1)$ and adult (dsGFP control) female locusts at these different time points. Supplementary Figures S6 and S7 show the temporal expression profiles for Halloween genes and ecdysone receptor components in ovaries of adult ( $d s G F P$ control) and adultoid (dsLmKr-h1) females, respectively.

In adult females, a significantly increased expression level was observed on day 12 (Ad D12) for $\mathrm{LmPhm}$, while a similar temporal trend was also noticeable for LmSpo and LmSad (Figure S6A,B,D). For $L m S h d$ and $L m E c R$ no significant changes in expression were observed (Figure S6E,F), while LmDib and $L m R X R$ levels seemed to progressively decline at different time points after the final molt (Figure S6C,G). No correlations were found between adult and adultoid locusts for the temporal profiles of ovarian LmKr-h1, LmSpo, LmPhm, LmDib, LmSad, LmShd, LmEcR and LmRXR transcripts.

In comparison with freshly molted adultoid females (Ao D0), LmPhm, LmEcR and LmRXR transcript levels were significantly reduced on day 4 (Ao D4) after the final molt (Figure S7B,F,G). Furthermore, in these adultoids the LmSpo, LmPhm, LmSad and LmEcR genes showed significantly increased transcript levels on day 8 (Ao D8) (Figure S7A,B,D,F). At this time point, the rise of LmSpo and $\mathrm{LmPhm}$ levels in adultoids appeared very pronounced, as on day 8 (D8) the transcript levels for both Halloween genes were also significantly higher than in adult females (Figure 5B,C). A similar temporal profile was noticed when investigating $L m K r-h 1$ expression levels in the ovaries of adultoid locusts (Figure S5C and Figure 5A). For LmDib no significant changes in expression were observed between the different time points analyzed in the adultoids (Figure S7C). Nevertheless, in comparison with adult (control) females, a significantly higher LmDib mRNA level was observed on day 12 (D12) (Figure 5D). In addition, when compared to control adults, significantly lower LmSpo, LmSad and LmRXR transcript levels were detected in the adultoids on day 4 (D4) (Figure 5B,E,H). No significant differences in $L m S h d$ and $L m E c R$ expression were observed between adult and adultoid females at the different time points (Figure $5 F, G$ ). 
Control Adult $\square$ dsLmKr-h1 Adultoid

\section{A}

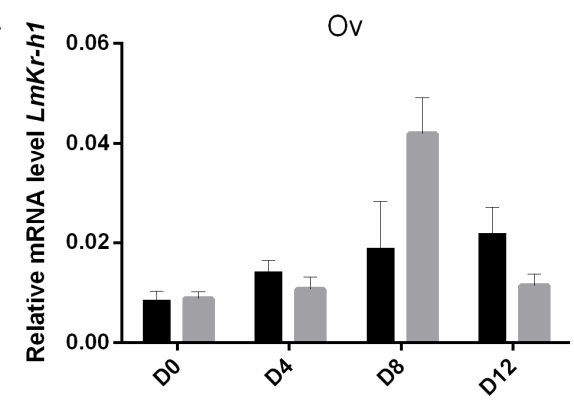

C

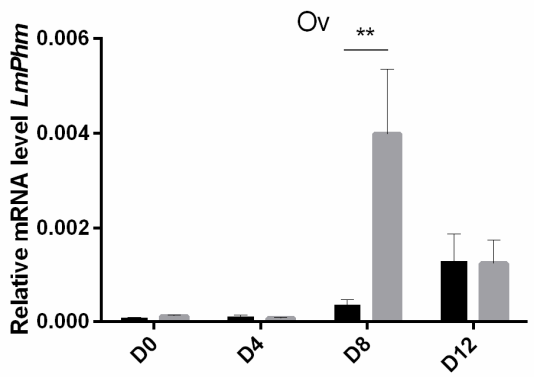

E

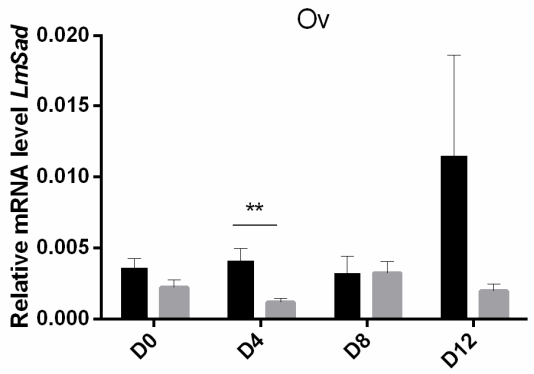

G

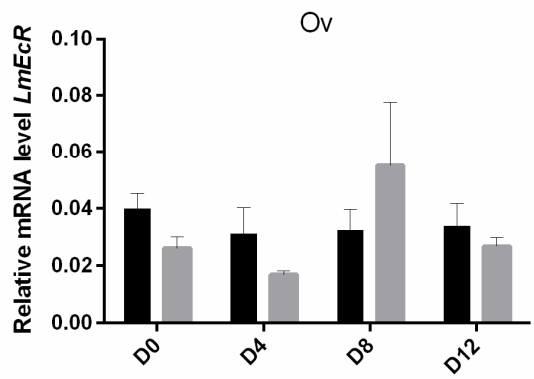

B

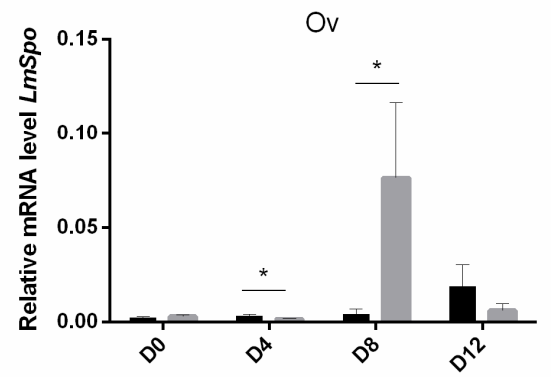

D

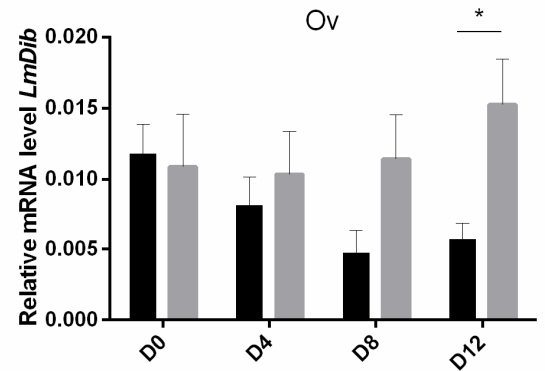

F

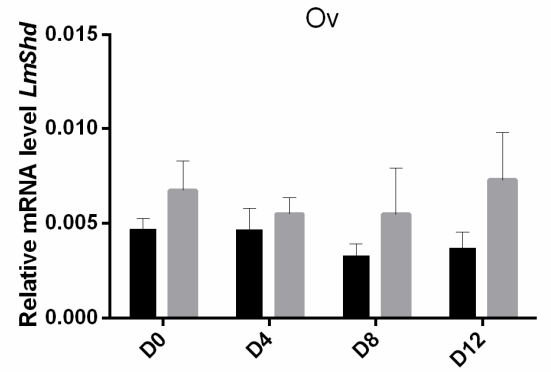

$\mathrm{H}$

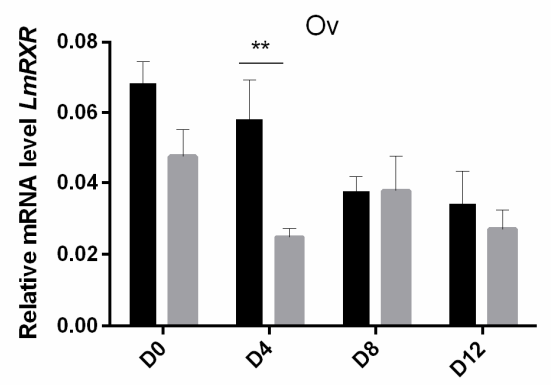

Figure 5. Relative transcript levels of $L m K r$ - $h 1$, several Halloween genes as well as $L m E c R$ and $L m R X R$ in ovaries of adult and adultoid L. migratoria females. Relative $L m K r$ - $h 1$ (A), Spook (LmSpo) (B), Phantom $(\mathrm{LmPhm})(\mathbf{C})$, Disembodied (LmDib) (D), Shadow (LmSad) (E), Shade (LmShd), (F), LmEcR (G) and LmRXR (H) transcript levels were measured in the ovaries of freshly molted (D0), 4-day-old (D4), 8-day-old (D8) and 12-day-old (D12) adultoid ( $d s L m K r-h 1)$ and adult (dsGFP: control) female locusts. The data represent mean \pm S.E.M. of four independent pools of three animals, run in duplicate and normalized to CG13220 and TubA1 transcript levels. Statistically significant differences between the measurements were found via a $t$-test on log-transformed data (with or without two-sided Welch's correction) and are indicated by (an) asterisk(s) $\left(^{*} p<0.05 ;{ }^{* *} p<0.01\right)$. With a Pearson correlation calculation, no significant correlations were found between transcript profiles of adult and adultoid locusts. 


\subsection{Adultoid Females Have Severe Defects in Mating and Egg Laying}

Mating, fecundity and fertility were assessed in the adultoid ( $d s L m K r-h 1)$ locusts in comparison with normal adults (dsGFP control). Therefore, the events of mating with a virgin adult male (showing an actual connection between the male and female genitals) and egg deposition were monitored for adult and adultoid females (Figure 6). We also registered the period between mating and oviposition, the incubation time from egg laying until hatching, as well as the numbers of eggs and hatchlings per egg pod that had been deposited by the females (Figure S8). Our results indicate that the mating process was significantly affected in the adultoid females. Adultoid females did not mate with virgin control males within the experimental timeframe (Figure 6A). In comparison, all control females mated successfully (Figure 6A). When paired with adultoid males, only a small fraction (14\%) of the adultoid females was mating (Figure 6A and Figure S9A-C and G-H). Oviposition was also severely impaired, since only the adultoid females that had mated also deposited their eggs (Figure 6B and Figure S9D-F). Moreover, they deposited their eggs significantly earlier than the adult females (Figure S8A). Interestingly, the eggs deposited by the adultoid females appeared similar in size and shape to the ones obtained from adult females. However, the incubation period between egg laying and hatching was significantly longer (Figure S8B). Supplementary Figure S8C shows the numbers of eggs and hatchlings per egg pod. Although adult and adultoid females did not significantly differ in the number of eggs they deposited per pod (fecundity), the number of hatchlings (fertility) was significantly lower for the offspring of adultoid locusts.
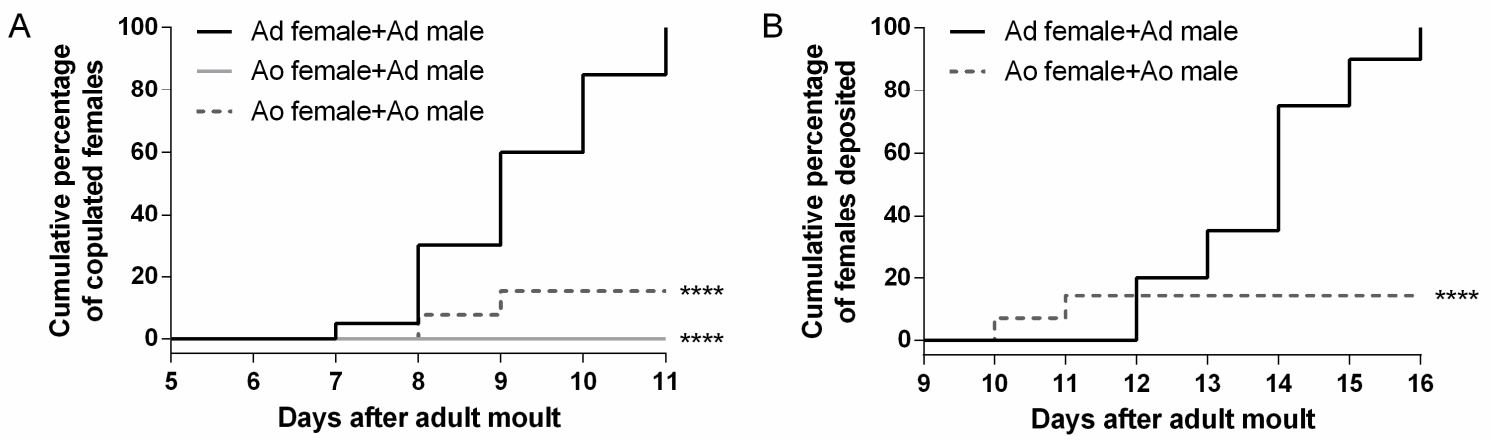

Figure 6. Mating and oviposition by adult and adultoid L. migratoria females. Mating and oviposition were observed for 20 adult (Ad) females (each combined with an adult male: 'Ad female + Ad male'), 20 adultoid (Ao) (each combined with an adult male: 'Ao female + Ad male') and 14 Ao (each combined with an adultoid male: 'Ao female + Ao male') females starting on day 5 of the adult (Ad) or adultoid (Ao) stage. (A) The cumulative percentage of mating females (achieving an actual connection between male and female genitals) and (B) the cumulative percentage of females that deposited eggs are shown. Since no mating was observed between adultoid females and adult males, the observation of oviposition was only made for the control condition, combining an adult female with an adult male, and the experimental condition, combining an adultoid female with an adultoid male. Statistically significant differences $(p)$ between the control and experimental conditions were found via a log-rank (Mantel-Cox) test and are indicated by asterisks $\left({ }^{* * * *} p<0.0001\right)$.

\section{Discussion}

\subsection{DsLmKr-h1 Injections in Fourth Nymphal Stage Induced the Developmental Transition to Adultoids}

The migratory locust, L. migratoria, is a hemimetabolan insect that normally passes five nymphal stages before the adult, reproductive stage is reached. The final molt is characterized by an incomplete metamorphosis process, which results in development of functional wings and reproductive organs. In this study, a final molt was precociously induced by RNAi-mediated knockdown of $L m K r-h 1$, a well-conserved transcription factor acting immediately downstream of the JH receptor Met, during the fourth nymphal stage (Figure 1). The adultoid phenotype resulting from this treatment displayed 
the coloration pattern that is typical for adults. On the other hand, its wings were not fully extended. Similar observations of precocious adult-like phenotypes were made after $K r-h 1$ knockdown in several other hemimetabolan insect species, such as B. germanica, R. prolixus, G. bimaculatus, N. lugens, C. lectularius and P. apterus $[10,11,13-17,27]$.

Locusts were injected with $d s L m K r-h 1$ on the day of their molt to the fourth instar and a second injection was given 3 days later. One day later (N4 D4), a significant downregulation of the $L m K r-h 1$ transcript level was detected in the fat body (Figure S3A). On the day of the adultoid molt (Ao D0), the $L m K r-h 1$ levels were clearly much higher than in the $d s G F P$-injected (control) N5 condition and resembled the normal adult levels (Ad D0) (Figure S3A), suggesting that the effect of the knockdown was temporally restricted to the N4 stage. Similar studies in the common bed bug, C. lectularius, and the German cockroach, B. germanica, showed similar efficiencies of $K r$ - $h 1$ transcript knockdown [14,17]. The majority (64\%) of the $d s L m K r$-h1-injected locusts developed into adultoids (Figure 1A and B). The presence of some fifth instar and intermediate phenotypes, as also observed by Lozano and Belles (2011) in B. germanica upon silencing $K r-h 1$, suggests some inter-individual variation in knockdown efficiency, which may account for the relatively mild reduction in the $L m K r-h 1$ transcript level that was measured in $d s L m K r$-h1-injected locusts on day 4 in the fourth nymphal stage [14]. A second injection in B. germanica was sufficient to obtain $100 \%$ adultoids, but this was not the case in the locusts of this study. The difficulty of obtaining adultoids with full efficiency $(100 \%)$ was also noticed in the common bed bug, C. lectularius. When knocking down $\mathrm{Kr}$ - $h 1$ in the penultimate instar, only $31 \%$ of the dsRNA-injected insects developed precociously into adultoids [17]. It is possible that the spatiotemporal characteristics of the RNAi-mediated knockdown influence its developmental outcome. In our study no downregulation of $L m K r-h 1$ was observed in the ovaries (Figure S3C), which can be explained by the previously described inefficient uptake of dsRNA into the follicle cells and oocytes [57,58]. Remarkably, a significantly increased $L m K r-h 1$ transcript level was detected in the CA (Figure S3B). This suggests the existence of a very dynamic and tissue-dependent regulation of this immediate early $\mathrm{JH}$ response gene, which may explain why its downregulation was not maintained longer and even resulted in a compensatory effect within the CA. Still, the phenotypic effects obtained in this experiment were very obvious and fully in line with the well-established role of $\mathrm{Kr}-\mathrm{h} 1 \mathrm{in}$ controlling the developmental timing of metamorphosis.

Expression of $K r-h 1$ is known to be controlled by JH activity, downstream of Met, as previously reported for several insect species $[8,10,12,14,59]$. Our qRT-PCR measurements indeed show that in dsGFP-injected control locusts, the expression of both $L m K r-h 1$ and LmMet in fat body was reduced on the day of molting to the fifth nymphal stage (N5 D0), followed by increased transcript levels of the adult specifier $\operatorname{LmE} 93$ on the next time point that was analyzed (N5 D3) (Figure S1A,D,G). These temporal profiles are in agreement with the described role of these factors in the control of metamorphosis. Interestingly, for the $d s L m K r$ - $h 1$-injected locusts that developed into adultoids, the total duration of the fourth nymphal stage (from N4 D0 until the N4-adultoid transition) was on average two days longer than that of a normal fourth nymphal stage (5 days) and thus similar to that of a normal fifth nymphal stage (7 days) (Figure 1C). Moreover, none of the adultoids and only a small fraction of the intermediate phenotypes resulting from the $L m K r-h 1$ knockdown had any further molts (Figure 1D). These observations indicate that the transition process from N4-nymph to adultoid can indeed be considered as a precocious metamorphosis, at which the locusts were reprogrammed to stop increasing their body size and start investing more energy and nutrients in reproduction. Furthermore, clear differences in transcript levels of MEKRE93 pathway components were observed during this time. Normally, the expression levels of the adult specifier gene E93 only start rising in the final nymphal stage, when JH titers drop and ecdysteroid titers rise, to ensure the final transition to the adult stage [21]. A significant rise in E93 levels was indeed observed on day three (N5 D3) in the control fifth nymphal locusts (Figure S1D,E). Therefore, the significantly higher LmE93 levels that were detected in the $d s L m K r-h 1$-injected locusts on day 4 of the fourth nymphal stage (Figure S3D,E) can explain the transition of the majority of these locusts into adultoids (Figure 1B). Ishimaru et al. (2019) 
also reported elevated $E 93$ transcript levels in premature G. bimaculatus adults after knocking down $K r-h 1$ [15]. A similar observation was made by Lozano and Belles (2011) who described that female fifth instar B. germanica, treated with $d s K r-h 1$, required on average two to three days more to perform the next molt [14]. LmMet expression measured in the fat body remained relatively stable on different time points during the fifth nymphal stage of control animals (Figure S1G). Similar temporal profiles were observed in B. germanica, N. lugens and P. apterus, with Met transcript levels staying relatively stable in nymphal whole body RNA samples, independently of JH titers [10-12,22]. However, in the CA of locusts, the LmMet levels appeared to gradually decline over time (Figure S1H). Interestingly, in freshly molted adultoids the transcript levels of MEKRE93 pathway components were more similar to the ones observed in adults than in fifth instar control locusts on the day of molting (Figure S3), emphasizing that the adult-like properties of the adultoid locusts were also noticeable at the molecular level.

\subsection{Comparison Between Adultoid and Adult Female Locusts during Their First Gonadotrophic Cycle}

In the current study, we mainly focus on the reproductive physiology of the adultoid females and compare this with normal adult female locusts (dsGFP-injected control). In this context we also analyzed the transcript levels of several genes involved in hormone synthesis, signal transduction and vitellogenesis. Our data indicate that the adultoid females have the following characteristics:

\subsubsection{An Accelerated Ovarian Maturation}

The adultoid females were characterized by an accelerated entry into the vitellogenic stage of the first gonadotrophic cycle (Figure 2). The first gonadotrophic cycle in the ovaries of female locusts can be divided in five stages: (i) an early growth period; (ii) the previtellogenic stage; (iii) the vitellogenic stage; (iv) the choriogenic stage and (v) ovulation and oviposition [60,61]. It is clear from Figure 2 that the adultoid females entered the vitellogenic stage several days prior to the adult control locusts. This stage is characterized by patency of the follicular epithelium, allowing the passage of lipids and vitellogenins from the hemolymph to the developing basal oocytes, causing them to grow. Although the basal oocytes in adultoid ovaries were slightly smaller on day four after the final molt, they became much larger than these in adults on the subsequent time points (D8 and D12 in Figure 2B; Figure 2D versus $2 \mathrm{~N}$ ). To further confirm this early incorporation of yolk material, transverse sections of the oocytes were made (Figure 2F-L). Whereas in oocytes of adultoid locusts, proteins (blue matrix) and lipid droplets (greyish droplets) were already visible on day 8 after the final molt (Figure 2G), it took oocytes of adult locusts approximately four additional days to enter this stage (Figure 2L). In addition, some adultoid ovaries had then already entered the second gonadotrophic cycle and also contained resorbing oocytes (Figure 2D-E', I-I'). When the eggshell or chorion was formed, the basal oocytes took on a shiny appearance, then ovulation occurred and fully grown oocytes entered the lateral oviducts (Figure 2E). Morphological analysis of these oocytes confirmed the presence of a chorion (Figure 2J).

\subsubsection{Associated Changes in Gene Expression Profiles in Fat Body and CA}

Vitellogenin synthesis was monitored at the mRNA level on different time points in both adult and adultoid female locusts. When compared to adult females, the fat body of adultoids contained significantly higher levels of both $L m V g 1$ and $L m V g 2$ on days eight and twelve after the final molt (D8 and D12 in Figure 3). This timing is fully in line with the observed increase in length of the basal oocytes occurring in the adultoid female ovaries (Figure 2B). In addition, significantly higher transcript levels of the immediate early JH response gene $L m K r-h 1$ were also detected in fat body of adultoid females compared to control adult females (Figure 4A). The importance of JH signaling in regulating female vitellogenesis was previously confirmed in the migratory locust, L. migratoria. A study by Song et al. (2014) described reduced $V g$ gene expression in the fat body, lipid accumulation in the primary oocytes, as well as a strong inhibition of oocyte maturation upon silencing LmMet and $L m K r-h 1$ [6]. These observations are corroborated by multiple other studies performed in numerous insect species $[13,23-25,27,29,31-34,38,62-64]$. In addition to the higher $L m K r$ - $h 1$ transcript levels 
detected in fat body of adultoid females (Figure S5A), lower LmE93 transcript levels were observed (Figure S5B), which may refer to the inhibitory effect of Kr-h1 on E93 expression [21,65]. LmE93 expression was extremely low on day 8 , when the adultoid females were in full vitellogenesis (D8 in Figure 4B). However, in fat body of normal adult females an inverse relationship between $L m K r-h 1$ and $L m E 93$ gene expression was not noticeable (Ad D0-D12 in Figure S1A,B), which may point at an important difference upstream in the regulation of these genes between adults and adultoids. In the CA, LmJHAMT and LmCYP15A1 expression levels were increased in the adult/adultoid stages, which may refer to the important roles that are played by JH in reproduction (Figure S1F,I; Figure S5F,I). Moreover, the relative expression level of $L m C Y P 15 A 1$ was higher on day 8 (Figure $4 \mathrm{H}$ ) in the vitellogenic adultoid females, when compared with the control adult females. Multiple studies also reported on the increased expression of $\mathrm{JH}$ biosynthetic enzymes during the gonadotrophic cycle of two different locust species [6,38], as well as several other insects [29,66-71]. During the maturation of reproductive organs and progression of the first gonadotrophic cycle, $\mathrm{JH}$ signaling is also crucial for the induction of polyploidy in the fat body cells, allowing for the massive metabolic conversion of stored energy and nutrients into yolk constituents, such as vitellogenins and lipids, which eventually will be incorporated into the basal oocytes during the vitellogenic phase $[63,64]$.

\subsubsection{Accelerated $K r-h 1$ and Halloween Gene Expression in the Ovary}

In addition to $\mathrm{JH}$, ecdysteroids are playing an important role in the reproductive physiology of female locusts. Ecdysteroid biosynthesis mainly takes place in the follicular cell layer of the developing oocytes [72,73]. In a series of enzymatic reactions involving the Halloween genes, the steroid hormone precursor cholesterol is converted to 20E [74]. When comparing female adultoids with control adults, significant differences in Halloween gene expression were observed on different time points after the final molt (Figure 5). In female adultoids, day 8 appeared to be a critical time point when a prominent peak of both $\mathrm{LmSpo}$ and $\mathrm{LmPhm}$ expression levels was observed (Figure S7A,B). Where Spo is considered to catalyze the rate-limiting step, $\mathrm{Phm}$ is important in the conversion of ketodiol to ketotriol [75-77]. In agreement with the current study (Figure S6), Marchal et al. (2011) also showed that the transcript levels of both Halloween genes were low in freshly molted adults and rose during the female reproductive cycle of the desert locust, S. gregaria [71]. The observed elevated LmSpo and $\mathrm{LmPhm}$ transcript levels in the ovaries of adultoids on day 8 after their final molt, coinciding with the significantly increased $K r-h 1$ levels in the same organs (Figure S5C), therefore once again reinforce our observations of an accelerated ovarian maturation in comparison with normal adult migratory locusts (D8 in Figure 5B,C). In addition to the accumulation of ecdysteroid conjugates in basal oocytes during vitellogenesis, acting as a source of ecdysteroids during embryonic development, ecdysteroid signaling also plays a role in choriogenesis, involving the heterodimeric nuclear receptor complex consisting of the ecdysone receptor (EcR) and the retinoid-X-receptor (RXR). An RNAi-mediated knockdown of $E c R / R X R$ in the desert locust, S. gregaria, and the Pacific beetle cockroach, D. punctata, resulted in females incapable of initiating choriogenesis; basal oocytes reached their maximal length, after which they were resorbed, thereby affecting ovulation as well $[49,78]$. These studies also suggested that EcR and RXR mediate critical ecdysteroid feedback to the CA, essential at the end of vitellogenesis for successful ovulation. In 1993, Belles et al. already suggested the existence of a crosstalk between $\mathrm{JH}$ and ecdysteroids in B. germanica, since ecdysteroids are responsible for the termination of the gonadotrophic cycle via lowering JH synthesis [79]. In our study, the ovarian LmEcR and LmRXR transcript levels did not differ significantly on the observed time points during the vitellogenic stage between adult and adultoid female locusts (D8 and D12 in Figure 5G,H). Nevertheless, in adultoid ovaries, the $L m E c R$ levels significantly increased at day eight, which may refer to the crucial role of ecdysteroid signaling in choriogenesis (Ao D8 in Figure S7F). These results can explain the normal appearance of the fully grown oocytes observed in adultoid females (Figure 2J). 


\subsubsection{Severe Defects in Mating and Egg Laying}

None of the adultoid females effectively mated with virgin control males (Figure 6A). Since differences in body size and anatomy between adultoid and adult locusts (Figure 1A and Figure S9B) could have an adverse effect on mating success, adultoid females were also paired with adultoid males (Figure S9A). In addition, in this setup, mating was negatively affected when compared to control adult locusts (Figure 6A). To verify whether adultoid males were capable of mating, some were also paired with control females, eventually confirming that mating was physically possible (Figure S9C). The few adultoid females that were able to mate needed fewer days to deposit their eggs (Figure 6B and Figure $\mathrm{S} 8 \mathrm{~A})$. However, it took the few hatchlings that were produced $(7.5 \%)$ several days longer to emerge (Figure S8B,C). Remarkably, all adultoid females, even if they did not mate, attempted to oviposit, but the unmated ones did not succeed and subsequently died in the coming days. Dissection of their abdomens revealed ovaries full of mature oocytes, some of them stuck in the oviducts and leaking yolk (Figure S9D,E). In L. migratoria, mating is known to induce physiological and endocrinological changes in the female resulting in appropriate maturation of the eggs and the subsequent deposition of fertile eggs in a suitable environment [80]. Mating in the kissing bug, R. prolixus, triggers the release of a myotropic peptide from the nervous system, resulting in ovulation and oviposition [81]. In Drosophila melanogaster, the Sex Peptide is present in the seminal fluid and transferred from male to female upon mating; in the female fly, it triggers a recently described neural circuitry that controls egg laying [82]. Therefore, the incapability of virgin adultoid L. migratoria females to oviposit might be due to the absence of such a physiological stimulus induced by mating. In addition, a recent study in the cricket, G. bimaculatus, where silencing $K r-h 1$ resulted in adultoid females, described the occurrence of an abnormal ovipositor morphology [15]. Although the ovipositors of the adultoid female locusts had a normal anatomical appearance, their size was smaller when compared to the adult ones (Figure S9F). Since the eggs of adultoids had a similar size as the ones produced by adults, it is not unlikely that this may have caused great discomfort during oviposition. Finally, while the number of eggs per egg pod (fecundity) produced by the adultoid females was similar to that of adults, the number of hatchlings (fertility) was severely reduced. The reasons for this low fertility are not fully clear. We observed that adultoid males were eager to mate with an adultoid or adult female and noticed that their testes and accessory glands had a normal adult-like appearance (Figure S9G,H). However, we do not know how much sperm was transferred upon mating and how fertile it was. Therefore, the observed hatchlings either developed from fertilized eggs or were generated by parthenogenesis $[83,84]$. Future dissections of adultoid male testes and adultoid female spermatheca, to verify the presence and transfer of sperm, would probably enable a more comprehensive interpretation of these data. Nevertheless, the fact that some eggs successfully hatched indicates that some adultoids were capable of generating offspring.

\section{Materials and Methods}

\subsection{Rearing of Animals}

The migratory locusts (L. migratoria) were reared under crowded conditions in cages that were situated in a room with controlled temperature $\left(32 \pm 1^{\circ} \mathrm{C}\right.$ ) and day/night cycle (photophase of $13 \mathrm{~h}$ ) at an ambient relative humidity between $40 \%$ and $60 \%$. The animals were fed freshly sprouted wheat leaves and dry oat flakes ad libitum. Pots containing a slightly moistened mixture of sand and turf were placed in the breeding cages allowing mated females to deposit their eggs. These pots were collected once a week and transferred to clean cages where eggs were allowed to hatch into first instar locusts.

\subsection{Tissue Collection}

The tissues of interest were dissected under a binocular microscope in L. migratoria Ringer solution (1 L: $9.82 \mathrm{~g} \mathrm{NaCl} ; 0.32 \mathrm{~g} \mathrm{CaCl}_{2} ; 0.48 \mathrm{~g} \mathrm{KCl} ; 0.73 \mathrm{~g} \mathrm{MgCl}_{2} ; 0.25 \mathrm{~g} \mathrm{NaHCO}_{3} ; 0.19 \mathrm{~g} \mathrm{NaH}_{2} \mathrm{PO}_{4} ; \mathrm{pH}$ 6.5) and snap-frozen by pooling them in MagNA Lyser Green Beads Tubes (Roche, Mannheim, Germany) or RNase-free Screw Cap Microcentrifuge tubes which were placed in liquid nitrogen to prevent RNA 
degradation. Tissues were collected in four pools of three animals each and were used in an RNA extraction protocol or stored at $-80^{\circ} \mathrm{C}$ until further processing.

\subsection{RNA Extraction and cDNA Synthesis}

Fat body and ovary samples of female migratory locusts were pooled in MagNA Lyser Green Beads Tubes (Roche) and homogenized using a MagNA Lyser instrument (30 sec, $5000 \mathrm{rpm}$; Roche). Total RNA was extracted using the RNeasy Lipid Tissue Kit (Qiagen, Austin, TX, USA) according to the manufacturer's protocol. An on-column DNase digestion (RNase-free DNase set, Qiagen) was performed to prevent genomic DNA contamination. The CA were pooled in RNase-free Screw Cap Microcentrifuge tubes. According to the manufacturer's protocol, total RNA was extracted using the RNAqueous-Micro Kit (Ambion; Life Technologies, Carlsbad, CA, USA), a specialized kit for total RNA extraction of small sized tissues. A DNase incubation step was included here as well. Subsequently, the quality and concentration of the resulting RNA samples were measured using a Nanodrop spectrophotometer (NanoPhotometer N60, Implen, München, Germany). From each RNA sample equal amounts ( $150 \mathrm{ng}$ for fat body and ovaries and $50 \mathrm{ng}$ for $\mathrm{CA}$ ) were reverse-transcribed using a mix of random hexamers and oligo(dT) primers according to the manufacturer's protocol (PrimeScript ${ }^{\mathrm{TM}}$ RT Reagent Kit, TaKaRa, Invitrogen Life Technologies, Carlsbad, CA, USA). The obtained cDNA was then diluted ten-fold with Milli-Q water (Merck Millipore, Darmstadt, Germany).

\subsection{Quantitative Real-Time PCR}

QRT-PCR primers for the different target genes (Supplementary Table S1) were designed using Primer Express software (Applied Biosystems, Foster City, CA, USA). These primer pairs were validated by designing relative standard curves for gene transcripts with serial (5x) dilutions of appropriate cDNA samples. The correlation coefficient and efficiency of the qRT-PCR reaction were measured $\left(\mathrm{R}^{2}\right.$ $=0.995-1 ; \mathrm{Eff} \%=90 \%-110 \%$ ) for each primer pair. All qRT-PCR reactions were performed in duplicate in 96-well plates on a StepOne Plus System (ABI Prism, Applied Biosystems, Foster City, CA, USA) according to the Fast SYBR Green PCR Master Mix protocol. Each reaction contained $5.0 \mu \mathrm{L}$ of fast SYBR Green, $0.5 \mu \mathrm{L}$ of each Forward and Reverse primer $(10 \mu \mathrm{M}), 1.5 \mu \mathrm{L}$ of Milli-Q water and $2.5 \mu \mathrm{L}$ of cDNA. "No template control" reactions were included to confirm absence of contamination; RT' reactions were performed to check for possible genomic DNA contamination. The following thermal cycling profile was used: $95^{\circ} \mathrm{C}$ for $10 \mathrm{~min}$, followed by 40 cycles of $95^{\circ} \mathrm{C}$ for $15 \mathrm{~s}$ and $60^{\circ} \mathrm{C}$ for $60 \mathrm{~s}$. Afterwards, a melt curve analysis was performed to confirm the specificity of the qRT-PCR reactions. Additionally, the amplification products were analyzed using horizontal agarose gel electrophoresis (1.2\% agarose gel containing GelRed ${ }^{\mathrm{TM}}$, Biotium, Fremont, CA, USA) and visualized under UV light. Only a single band of the expected size for each transcript was observed, further cloned and sequenced (TOPO ${ }^{\circledR}$ TA Cloning Kit for sequencing, Invitrogen, Carlsbad, CA, USA) to confirm target specificity.

Optimal housekeeping genes were selected using geNorm software [85]. In the designed experiment rps13 + CG13220, CG13220 + TubA1 and rp49 + rps13 appeared to be most stable in the studied fat body, ovary and CA samples, respectively. Relative expression levels were determined according to the comparative $\mathrm{Ct}$ method $(\Delta \Delta \mathrm{Ct})$ [85]. QRT-PCR was used to determine the temporal distributions of the transcripts of interest. Moreover, statistically significant differences between the $d s L m K r-h 1$ and $d s G F P$ (control)-injected female locusts were found via a $t$-test on the log transformed data (with or without two-sided Welch's correction) using GraphPad Prism 6 (GraphPad Software Inc., San Diego, CA, USA). The correlation coefficient (r) between the transcript profiles of adult and adultoid locusts was obtained by a Pearson correlation calculation.

\subsection{RNA Interference Experiments}

Double-stranded (ds)RNA constructs for $L m K r$ - $h 1$ and GFP (control) were produced using Ambion's MEGAScript RNAi kit following the manufacturer's protocol (Figure S2). This procedure is based on a high-yield in vitro transcription reaction from a user-provided DNA template with a 
T7 promoter sequence at the $5^{\prime}$ ends of each strand. Forward and reverse primers flanked by the T7 promoter sequence (given in Supplementary Table S2) were used in a PCR reaction with REDTaq DNA polymerase (Sigma-Aldrich, Darmstadt, Germany) to amplify a fragment of the target gene. The resulting amplicons were analyzed using horizontal agarose gel electrophoresis $(1.2 \%$ agarose gel containing GelRed ${ }^{\mathrm{TM}}$, Biotium, Fremont, CA, USA) and visualized under UV light. Only a single band was observed, which was subsequently cloned and sequenced (TOPO ${ }^{\circledR}$ TA Cloning Kit for sequencing, Invitrogen, Carlsbad, CA, USA) to confirm target specificity. The verified constructs were used as template in a high-yield in vitro transcription reaction with T7 RNA polymerase. Remaining ssRNA and DNA were removed in a nuclease digestion step. Quality and concentration of the produced dsRNA were determined using a Nanodrop spectrophotometer (NanoPhotometer N60, Implen, München, Germany). To confirm dsRNA integrity, a small amount of the reaction product was checked by horizontal agarose gel electrophoresis (1.2\% agarose gel containing GelRed ${ }^{\mathrm{TM}}$, Biotium, Fremont, CA, USA).

83 Newly molted fourth nymphal (N4) female locusts, synchronized on the day of ecdysis (D0), were injected with $6 \mu \mathrm{L}$ dsRNA against $L m K r-h 1$ (dsLmKr-h1) (400 ng dsRNA/locust, diluted in L. migratoria Ringer). A second injection was given on day three of the fourth nymphal stage (N4 D3) to ensure an efficient knockdown of $L m K r-h 1$ during this nymphal stage. As a control condition, another group of 183 locusts was injected with dsRNA against GFP (dsGFP) following the same injection scheme. In our L. migratoria colony, we previously observed that the penultimate (N4) and final (N5) nymphal stage are lasting for 5 and 7 days, respectively. Female adult locusts are in the vitellogenic stage of their first gonadotrophic cycle 12 days (D12) after their final molt. As such, several time points during these developmental stages were chosen for tissue collection: day 4 for fourth nymphal female locusts (N4 D4), day 0 (N5 D0), 3 (N5 D3) and 6 (N5 D6) for fifth nymphal control locusts and day 0 (D0), 4 (D4), 8 (D8) and 12 (D12) for the adult (Ad) and adultoid (Ao) locusts.

\subsection{Observing Ecdysis}

The ecdysis of $83 d s L m K r-h 1$ and $183 d s G F P$-injected female locusts was closely observed. Starting from fourth nymphal locusts, synchronized on the day of ecdysis, the duration and type of each developmental stage were monitored in detail. Data were analyzed with a log-rank (Mantel-Cox) test using GraphPad Prism 6 (GraphPad Software Inc., San Diego, CA, USA).

\subsection{Measurement of Oocyte Length}

Oocytes at the base of the ovarioles, were carefully removed from the dissected ovaries of adult ( $d s G F P$ control) and adultoid ( $d s K r-h 1)$ female locusts. Oocyte length was measured using millimeter graph paper. For 12 locusts per condition, the average size of three basal oocytes was calculated. Data were analyzed with a nonparametric Mann-Whitney test using GraphPad Prism 6 (GraphPad Software Inc., San Diego, CA, USA).

\subsection{Microscopy and Histological Analysis}

Images of randomly chosen ovaries and ovarioles dissected from adult ( $d s G F P$ control) and adultoid (dsLmKr-h1) females were obtained with a light microscope (Zeiss SteREO Discovery V8, Oberkochen, Germany) equipped with an AxioCam ICc3 camera using the AxioVision 4.7 (Carl Zeiss-Benelux, Oberkochen, Germany).

Oocyte sections were made according to Billen (2006) [86]. In brief, the dissected oocytes were fixed in $2 \%$ glutaraldehyde for 12 to $24 \mathrm{~h}$ where after the glutaraldehyde was replaced by sodium cacodylate buffer and postfixed in $2 \%$ osmium tetraoxide. Next, the samples were dehydrated in a graded acetone series and embedded in araldite. Semi-thin $(1 \mu \mathrm{m})$ sections, obtained using a microtome (Leica EM UC6 microtome, Nussloch, Germany), were stained with methylene blue and thionine. The resulting oocyte sections were visualized using a light microscope (Zeiss Axio Imager Z1, Oberkochen, Germany) 
equipped with an AxioCam MRm camera (1388 × 1040 pixels) and the software program Zen 2012 (Bleu Edition: Carl Zeiss-Benelux, Oberkochen, Germany).

\subsection{Observation of Mating, Egg Deposition and Hatching}

Mating and oviposition events were monitored in adult (dsGFP control) and adultoid (dsLmKr-h1) female locusts, which were obtained in a follow-up experiment using the same injection scheme as described in $\$ 2.5$. In this context, the following combinations were studied: 20 control males $\times$ control females, 20 control males $\times$ adultoid females and 14 adultoid males $\times$ adultoid females. On day 4 of the locusts' adult/adultoid life, the female locusts were separated from the males and transferred to individual cages where they were fed daily with freshly sprouted wheat leaves. On day 5 of the locusts' adult/adultoid life, one sexually mature virgin male was introduced to each female and mating (i.e., actual connection between the male and female genitals) was monitored. If mating was observed within two hours of the male's introduction, the male was allowed to stay with the female for 24 h. Pots filled with a slightly humidified sand/turf mixture were supplied to the females to allow oviposition. These pots were checked daily for egg pods. The total number of deposited eggs per egg pod was counted to analyze fecundity. The percentage of hatchlings was determined to analyze fertility. If no mating was observed within two hours of the male's introduction, the male was removed, and another mating attempt was made the following day using a different virgin male. A log-rank (Mantel-Cox) test in GraphPad Prism 6 (GraphPad Software Inc., San Diego, CA, USA) was used to compare mating and egg deposition between adult and adultoid female locusts. Statistically significant differences in days between mating and egg laying, the number of eggs laid, days between egg laying and hatching and the number and percentage of hatchlings between the different conditions were found via a nonparametric Mann-Whitney test using GraphPad Prism 6 (GraphPad Software Inc., San Diego, CA, USA).

\section{Conclusions}

In conclusion, our findings show that the knockdown of $L m K r-h 1$ in the fourth instar resulted in an adultoid phenotype characterized by precocious development of adult features, an accelerated ovarian maturation and reduced mating. Only the few adultoid females capable of mating were also able to deposit their eggs successfully. Although the fecundity of the adultoid females did not seem to differ from that of control adults, the percentage of hatchlings was much lower. When taking into account the major problems that are still caused by migrating locust swarms in many countries worldwide, RNAi-based and/or pharmacological strategies targeting JH signaling pathway components that proved to be crucial for locust development and reproduction would probably be very effective means to control locust population growth, swarm formation and expansion.

Supplementary Materials: The Supplementary Materials can be found at http://www.mdpi.com/1422-0067/21/17/ 6058/s1.

Author Contributions: Conceptualization, M.G. and E.M.; data curation, M.G.; formal analysis, M.G.; funding acquisition, J.V.B.; investigation, M.G., S.S., T.S. and E.B.; methodology, M.G.; project administration, J.V.B.; resources, J.V.B.; supervision, J.V.B.; validation, M.G. and J.V.B.; visualization, M.G.; writing—original draft, M.G., E.M. and J.V.B.; writing - review and editing, M.G., S.S., T.S., E.B., E.M. and J.V.B. All authors have read and agreed to the published version of the manuscript.

Funding: This work was supported by grants from the Special Research Fund of KU Leuven (C14/15/050; C14/19/069), the European Union's Horizon 2020 Research and Innovation programme (No. 634361), and the Research Foundation of Flanders (FWO) (G0F2417N; G090919N) to J.V.B.. M.G. and S.S. obtained a PhD fellowship from FWO (1S48516N to M.G.; 1S42119N to S.S.).

Acknowledgments: The authors gratefully thank Evelien Herinckx for taking care of the locust breeding and Johan Billen and An Vandoren for their help with the histological sections. The authors are also grateful to Cynthia Lenaerts for her valuable advice regarding our study of the adultoid phenotype.

Conflicts of Interest: The authors declare no conflict of interest. 


\section{Abbreviations}

\begin{tabular}{|c|c|}
\hline $20 \mathrm{E}$ & 20-hydroxyecdysone \\
\hline Ad & Adult \\
\hline Ao & Adultoid \\
\hline bHLH & Basic helix-loop-helix \\
\hline CA & Corpora allata \\
\hline cDNA & Complementary DNA \\
\hline CYP15A1 & Methyl farnesoate epoxidase \\
\hline $\mathrm{D}$ & Day \\
\hline $\mathrm{Dib}$ & Disembodied \\
\hline DNA & Deoxyribonucleic acid \\
\hline ds & Double-stranded \\
\hline dsRNA & Double-stranded RNA \\
\hline E93 & Ecdysone-induced protein 93 \\
\hline EcR & Ecdysone receptor \\
\hline GFP & Green fluorescent protein \\
\hline $\mathrm{JH}$ & Juvenile hormone \\
\hline JHAMT & Juvenile hormone acid O-methyltransferase \\
\hline Kr-h1 & Krüppel-homolog 1 \\
\hline MEKRE93 & Methoprene-tolerant-Krüppel homolog 1-E93 \\
\hline Met & Methoprene-tolerant \\
\hline mRNA & Messenger RNA \\
\hline $\mathrm{N}$ & Nymph \\
\hline PAS & Per-Arnt-Sim \\
\hline PCR & Polymerase chain reaction \\
\hline Phm & Phantom \\
\hline qRT-PCR & Quantitative reverse transcription (real-time) PCR \\
\hline RNA & Ribonucleic acid \\
\hline RNAi & RNA interference \\
\hline RP49 & Ribosomal protein 49 \\
\hline RXR & Retinoid-X-receptor \\
\hline Sad & Shadow \\
\hline Shd & Shade \\
\hline Spo & Spook \\
\hline Tai & Taiman \\
\hline TubA1 & Tubulin A1 \\
\hline USP & Ultraspiracle \\
\hline $\mathrm{Vg}$ & Vitellogenin \\
\hline
\end{tabular}

\section{References}

1. Riddiford, L.M. How does juvenile hormone control insect metamorphosis and reproduction? Gen. Comp. Endocrinol. 2012, 179, 477-484. [CrossRef]

2. Hill, R.J.; Billas, I.M.L.; Bonneton, F.; Graham, L.D.; Lawrence, M.C. Ecdysone Receptors: From the Ashburner Model to Structural Biology. Annu. Rev. Entomol. 2013, 58, 251-271. [CrossRef]

3. Wilson, T.G.; Fabian, J. A Drosophila melanogaster mutant resistant to a chemical analog of juvenile hormone. Dev. Biol. 1986, 118, 190-201. [CrossRef]

4. Charles, J.-P.; Iwema, T.; Epa, V.C.; Takaki, K.; Rynes, J.; Jindra, M. Ligand-binding properties of a juvenile hormone receptor, Methoprene-tolerant. Proc. Natl. Acad. Sci. USA 2011, 108, 21128-21133. [CrossRef]

5. Godlewski, J.; Wang, S.; Wilson, T.G. Interaction of bHLH-PAS proteins involved in juvenile hormone reception in Drosophila. Biochem. Biophys. Res. Commun. 2006, 342, 1305-1311. [CrossRef] [PubMed]

6. Song, J.; Wu, Z.; Wang, Z.; Deng, S.; Zhou, S. Krüppel-homolog 1 mediates juvenile hormone action to promote vitellogenesis and oocyte maturation in the migratory locust. Insect Biochem. Mol. Biol. 2014, 52, 94-101. [CrossRef] [PubMed] 
7. Konopova, B.; Jindra, M. Juvenile hormone resistance gene Methoprene-tolerant controls entry into metamorphosis in the beetle Tribolium castaneum. Proc. Natl. Acad. Sci. USA 2007, 104, 10488-10493. [CrossRef] [PubMed]

8. Minakuchi, C.; Namiki, T.; Shinoda, T. Krüppel homolog 1, an early juvenile hormone-response gene downstream of Methoprene-tolerant, mediates its anti-metamorphic action in the red flour beetle Tribolium castaneum. Dev. Biol. 2009, 325, 341-350. [CrossRef]

9. Chafino, S.; Ureña, E.; Casanova, J.; Casacuberta, D.M.; Franch-Marro, X.; Martín, D. Upregulation of E93 Gene Expression Acts as the Trigger for Metamorphosis Independently of the Threshold Size in the Beetle Tribolium castaneum. Cell Rep. 2019, 27, 1039-1049.e2. [CrossRef]

10. Konopova, B.; Smykal, V.; Jindra, M. Common and Distinct Roles of Juvenile Hormone Signaling Genes in Metamorphosis of Holometabolous and Hemimetabolous Insects. PLoS ONE 2011, 6, e28728. [CrossRef]

11. Lozano-Fernandez, J.; Bellés, X. Role of Methoprene-Tolerant (Met) in Adult Morphogenesis and in Adult Ecdysis of Blattella germanica. PLoS ONE 2014, 9, e103614. [CrossRef]

12. Smykal, V.; Daimon, T.; Kayukawa, T.; Takaki, K.; Shinoda, T.; Jindra, M. Importance of juvenile hormone signaling arises with competence of insect larvae to metamorphose. Dev. Biol. 2014, 390, 221-230. [CrossRef] [PubMed]

13. Villalobos-Sambucaro, M.J.; Riccillo, F.L.; Calderón-Fernández, G.M.; Sterkel, M.; Diambra, L.; Ronderos, J.R. Genomic and functional characterization of a methoprene-tolerant gene in the kissing-bug Rhodnius prolixus. Gen. Comp. Endocrinol. 2015, 216, 1-8. [CrossRef] [PubMed]

14. Lozano-Fernandez, J.; Bellés, X. Conserved repressive function of Krüppel homolog 1 on insect metamorphosis in hemimetabolous and holometabolous species. Sci. Rep. 2011, 1, 163. [CrossRef]

15. Ishimaru, Y.; Tomonari, S.; Watanabe, T.; Noji, S.; Mito, T. Regulatory mechanisms underlying the specification of the pupal-homologous stage in a hemimetabolous insect. Philos. Trans. R. Soc. B: Biol. Sci. 2019, 374, 20190225. [CrossRef]

16. Li, K.; Yuan, S.Y.; Nanda, S.; Wang, W.X.; Lai, F.X.; Fu, Q.; Wan, P.J. The Roles of E93 and Kr-h1 in Metamorphosis of Nilaparvata lugens. Front. Physiol. 2018, 9, 1677. [CrossRef]

17. Gujar, H.; Palli, S.R. Krüppel homolog 1 and E93 mediate Juvenile hormone regulation of metamorphosis in the common bed bug, Cimex lectularius. Sci. Rep. 2016, 6, 26092. [CrossRef]

18. Jin, M.-N.; Xue, J.; Yao, Y.; Lin, X.-D. Molecular Characterization and Functional Analysis of Krüppel-homolog 1 (Kr-h1) in the Brown Planthopper, Nilaparvata lugens (Stål). J. Integr. Agric. 2014, 13, 1972-1981. [CrossRef]

19. Ureña, E.; Manjón, C.; Franch-Marro, X.; Martín, D. Transcription factor E93 specifies adult metamorphosis in hemimetabolous and holometabolous insects. Proc. Natl. Acad. Sci. USA 2014, 111, 7024-7029. [CrossRef]

20. Ureña, E.; Chafino, S.; Manjón, C.; Franch-Marro, X.; Martín, D. The Occurrence of the Holometabolous Pupal Stage Requires the Interaction between E93, Krüppel-Homolog 1 and Broad-Complex. PLoS Genet. 2016, 12, e1006020. [CrossRef]

21. Bellés, X. Krüppel homolog 1 and E93: The doorkeeper and the key to insect metamorphosis. Arch. Insect Biochem. Physiol. 2019, 103, e21609. [CrossRef] [PubMed]

22. Lin, X.; Yao, Y.; Wang, B. Methoprene-tolerant (Met) and Krüpple-homologue 1 (Kr-h1) are required for ovariole development and egg maturation in the brown plant hopper. Sci. Rep. 2015, 5, 18064. [CrossRef] [PubMed]

23. Gujar, H.; Palli, S.R. Juvenile hormone regulation of female reproduction in the common bed bug, Cimex lectularius. Sci. Rep. 2016, 6, 35546. [CrossRef] [PubMed]

24. Liu, W.; Li, Y.; Zhu, L.; Zhu, F.; Lei, C.; Wang, X.-P. Juvenile hormone facilitates the antagonism between adult reproduction and diapause through the methoprene-tolerant gene in the female Colaphellus bowringi. Insect Biochem. Mol. Biol. 2016, 74, 50-60. [CrossRef] [PubMed]

25. Marchal, E.; Hult, E.F.; Huang, J.; Pang, Z.; Stay, B.; Tobe, S.S. Methoprene-Tolerant (Met) Knockdown in the Adult Female Cockroach, Diploptera punctata Completely Inhibits Ovarian Development. PLoS ONE 2014, 9, e106737. [CrossRef] [PubMed]

26. Zou, Z.; Saha, T.T.; Roy, S.; Shin, S.W.; Backman, T.W.H.; Girke, T.; White, K.P.; Raikhel, A.S. Juvenile hormone and its receptor, methoprene-tolerant, control the dynamics of mosquito gene expression. Proc. Natl. Acad. Sci. USA 2013, 110, E2173-E2181. [CrossRef] 
27. Smykal, V.; Bajgar, A.; Provazník, J.; Fexova, S.; Buricova, M.; Takaki, K.; Hodkova, M.; Jindra, M.; Dolezel, D. Juvenile hormone signaling during reproduction and development of the linden bug, Pyrrhocoris apterus. Insect Biochem. Mol. Biol. 2014, 45, 69-76. [CrossRef]

28. Bilen, J.; Atallah, J.; Azanchi, R.; Levine, J.D.; Riddiford, L.M. Regulation of onset of female mating and sex pheromone production by juvenile hormone in Drosophila melanogaster. Proc. Natl. Acad. Sci. USA 2013, 110, 18321-18326. [CrossRef]

29. Parthasarathy, R.; Sun, Z.; Bai, H.; Palli, S.R. Juvenile hormone regulation of vitellogenin synthesis in the red flour beetle, Tribolium castaneum. Insect Biochem. Mol. Biol. 2010, 40, 405-414. [CrossRef]

30. Sheng, Z.; Xu, J.; Bai, H.; Zhu, F.; Palli, S.R. Juvenile Hormone Regulates Vitellogenin Gene Expression through Insulin-like Peptide Signaling Pathway in the Red Flour Beetle, Tribolium castaneum. J. Biol. Chem. 2011, 286, 41924-41936. [CrossRef]

31. Ma, L.; Zhang, W.-N.; Liu, C.; Chen, L.; Xu, Y.; Xiao, H.; Liang, G. Methoprene-Tolerant (Met) Is Indispensable for Larval Metamorphosis and Female Reproduction in the Cotton Bollworm Helicoverpa armigera. Front. Physiol. 2018, 9, 1-12. [CrossRef] [PubMed]

32. Naghdi, M.; Maestro, J.-L.; Belles, X.; Bandani, A. Transduction of the vitellogenic signal of juvenile hormone by Methoprene-tolerant in the cockroach Blattella germanica (L.) (Dictyoptera, Blattellidae). Arthropods 2016, 5, 130-136.

33. Saiki, R.; Gotoh, H.; Toga, K.; Miura, T.; Maekawa, K. High juvenile hormone titre and abdominal activation of JH signalling may induce reproduction of termite neotenics. Insect Mol. Biol. 2015, 24, 432-441. [CrossRef] [PubMed]

34. Wang, J.-L.; Saha, T.T.; Zhang, Y.; Zhang, C.; Raikhel, A.S. Juvenile hormone and its receptor methoprene-tolerant promote ribosomal biogenesis and vitellogenesis in the Aedes aegypti mosquito. J. Biol. Chem. 2017, 292, 10306-10315. [CrossRef] [PubMed]

35. Wang, X.; Hou, Y.; Saha, T.T.; Pei, G.; Raikhel, A.S.; Zou, Z. Hormone and receptor interplay in the regulation of mosquito lipid metabolism. Proc. Natl. Acad. Sci. USA 2017, 114, E2709-E2718. [CrossRef] [PubMed]

36. Tang, Y.; He, H.; Qu, X.; Cai, Y.; Ding, W.; Qiu, L.; Li, Y. RNA interference-mediated knockdown of the transcription factor Krüppel homologue 1 suppresses vitellogenesis in Chilo suppressalis. Insect Mol. Biol. 2019, 29, 183-192. [CrossRef]

37. Wang, Z.; Yang, L.; Song, J.; Kang, L.; Zhou, S. An isoform of Taiman that contains a PRD-repeat motif is indispensable for transducing the vitellogenic juvenile hormone signal in Locusta migratoria. Insect Biochem. Mol. Biol. 2017, 82, 31-40. [CrossRef]

38. Gijbels, M.; Lenaerts, C.; Vanden Broeck, J.; Marchal, E. Juvenile Hormone receptor Met is essential for ovarian maturation in the Desert Locust, Schistocerca gregaria. Sci. Rep. 2019, 9, 10797. [CrossRef]

39. Wyatt, G.R.; Braun, R.P.; Zhang, J. Priming effect in gene activation by juvenile hormone in locust fat body. Arch. Insect Biochem. Physiol. 1996, 32, 633-640. [CrossRef]

40. Davey, K.G.; Sevala, V.L.; Gordon, D.R. The action of juvenile hormone and antigonadotropin on the follicle cells of Locusta migratoria. Invertebr. Reprod. Dev. 1993, 24, 39-45. [CrossRef]

41. Jing, Y.-P.; An, H.; Zhang, S.; Ningbo, W.; Shutang, Z. Protein kinase C mediates juvenile hormone-dependent phosphorylation of $\mathrm{Na}+\mathrm{K}+$-ATPase to induce ovarian follicular patency for yolk protein uptake. J. Biol. Chem. 2018, 293, 20112-20122. [CrossRef] [PubMed]

42. Sevala, V.L.; Davey, K.G. Action of juvenile hormone on the follicle cells ofRhodnius prolixus: Evidence for a novel regulatory mechanism involving protein kinase C. Cell. Mol. Life Sci. 1989, 45, 355-356. [CrossRef]

43. Sevala, V.L.; Davey, K.; Prestwich, G.D. Photoaffinity labeling and characterization of a juvenile hormone binding protein in the membranes of follicle cells of Locusta migratoria. Insect Biochem. Mol. Biol. 1995, 25, 267-273. [CrossRef]

44. Seidelmann, K.; Helbing, C.; Göbeler, N.; Weinert, H. Sequential oogenesis is controlled by an oviduct factor in the locusts Locusta migratoria and Schistocerca gregaria: Overcoming the doctrine that patency in follicle cells is induced by juvenile hormone. J. Insect Physiol. 2016, 90, 1-7. [CrossRef] [PubMed]

45. Lanot, R.; Thiebold, J.; Lagueux, M.; Goltzené, F.; Hoffmann, J.A. Involvement of ecdysone in the control of meiotic reinitiation in oocytes of Locusta migratoria (Insecta, orthoptera). Dev. Biol. 1987, 121, 174-181. [CrossRef]

46. Isaac, R.E.; Rees, H.H. Isolation and identification of ecdysteroid phosphates and acetylecdysteroid phosphates from developing eggs of the locust, Schistocerca gregaria. Biochem. J. 1984, 221, 459-464. [CrossRef] 
47. Tawfik, A.I.; Vedrová, A.; Sehnal, F. Ecdysteroids during ovarian development and embryogenesis in solitary and gregarious Schistocerca gregaria. Arch. Insect Biochem. Physiol. 1999, 41, 134-143. [CrossRef]

48. Lagueux, M.; Harry, P.; Hoffmann, J.A. Ecdysteroids are bound to vitellin in newly laid eggs of locusta. Mol. Cell. Endocrinol. 1981, 24, 325-338. [CrossRef]

49. Lenaerts, C.; Marchal, E.; Peeters, P.; Vanden Broeck, J. The ecdysone receptor complex is essential for the reproductive success in the female desert locust, Schistocerca gregaria. Sci. Rep. 2019, 9, 15. [CrossRef]

50. Chapuis, M.-P.; Loiseau, A.; Michalakis, Y.; Lecoq, M.; Franc, A.; Estoup, A. Outbreaks, gene flow and effective population size in the migratory locust, Locusta migratoria: A regional-scale comparative survey. Mol. Ecol. 2009, 18, 792-800. [CrossRef]

51. Zhang, L.; Lecoq, M.; Latchininsky, A.; Hunter, D. Locust and Grasshopper Management. Annu. Rev. Entomol. 2019, 64, 15-34. [CrossRef] [PubMed]

52. Cullen, D.A.; Cease, A.J.; Latchininsky, A.V.; Ayali, A.; Berry, K.; Buhl, J.; De Keyser, R.; Foquet, B.; Hadrich, J.C.; Matheson, T.; et al. From Molecules to Management: Mechanisms and Consequences of Locust Phase Polyphenism. Adv. Insect Physiol. 2017, 53, 167-285. [CrossRef]

53. Food and Agriculture Organization of the United Nations. Madagascar Locust Crisis. Food and Agriculture Organization of the United Nations. Available online: http://www.fao.org/emergencies/crisis/madagascarlocust (accessed on 24 July 2020).

54. Roussi, A. The Battle to contain gigantic locust swarms. Nature 2020, 576, 330. [CrossRef] [PubMed]

55. Stephen, S. 'Biblical' swarm of locusts plague Italian farmlands in worst infestation since WWII. Fox News. Available online: https://www.foxnews.com/world/biblical-locusts-italy-sardinia-farmers (accessed on 24 July 2020).

56. Carlone, M.; Sestito, D. 'There's nothing left' - Sardinian farmland stripped by locust swarms. CGTN. Available online: https://newseu.cgtn.com/news/2020-06-25/-There-s-nothing-left-Sardinian-farmlandstripped-by-locust-swarms-RzaYxkBVuw/index.html (accessed on 24 July 2020).

57. Ren, D.; Cai, Z.; Song, J.; Wu, Z.; Zhou, S. dsRNA uptake and persistence account for tissue-dependent susceptibility to RNA interference in the migratory locust, Locusta migratoria. Insect Mol. Biol. 2013, 23, 175-184. [CrossRef]

58. Wynant, N.; Verlinden, H.; Breugelmans, B.; Simonet, G.; Vanden Broeck, J. Tissue-dependence and sensitivity of the systemic RNA interference response in the desert locust, Schistocerca gregaria. Insect Biochem. Mol. Biol. 2012, 42, 911-917. [CrossRef]

59. Minakuchi, C.; Zhou, X.; Riddiford, L.M. Krüppel homolog 1 (Kr-h1) mediates juvenile hormone action during metamorphosis of Drosophila melanogaster. Mech. Dev. 2008, 125, 91-105. [CrossRef]

60. Peel, A.D.; Akam, M. The dynamics of yolk deposition in the desert locust Schistocerca gregaria. J. Insect Physiol. 2007, 53, 436-443. [CrossRef]

61. Tobe, S.S.; Pratt, G.E. Corpus allatum activity in vitro during ovarian maturation in the desert locust, Schistocerca gregaria. J. Exp. Biol. 1975, 62, 611-627.

62. Guo, W.; Wu, Z.; Yang, L.; Cai, Z.; Zhao, L.; Zhou, S. Juvenile hormone-dependent Kazal-type serine protease inhibitor Greglin safeguards insect vitellogenesis and egg production. FASEB J. 2018, 33, 917-927. [CrossRef]

63. Guo, W.; Wu, Z.; Song, J.; Jiang, F.; Wang, Z.; Deng, S.; Walker, V.K.; Zhou, S. Juvenile Hormone-Receptor Complex Acts on Mcm4 and Mcm7 to Promote Polyploidy and Vitellogenesis in the Migratory Locust. PLoS Genet. 2014, 10, e1004702. [CrossRef]

64. Wu, Z.; Guo, W.; Yang, L.; He, Q.; Zhou, S. Juvenile hormone promotes locust fat body cell polyploidization and vitellogenesis by activating the transcription of Cdk6 and E2f1. Insect Biochem. Mol. Biol. 2018, 102, 1-10. [CrossRef] [PubMed]

65. Mao, Y.; Li, Y.; Gao, H.; Lin, X. The Direct Interaction between E93 and Kr-h1 Mediated Their Antagonistic Effect on Ovary Development of the Brown Planthopper. Int. J. Mol. Sci. 2019, 20, 2431. [CrossRef] [PubMed]

66. Kinjoh, T.; Kaneko, Y.; Itoyama, K.; Mita, K.; Hiruma, K.; Shinoda, T. Control of juvenile hormone biosynthesis in Bombyx mori: Cloning of the enzymes in the mevalonate pathway and assessment of their developmental expression in the corpora allata. Insect Biochem. Mol. Biol. 2007, 37, 808-818. [CrossRef] [PubMed]

67. Mayoral, J.G.; Nouzova, M.; Yoshiyama, M.; Shinoda, T.; Hernandez-Martinez, S.; Dolghih, E.; Turjanski, A.G.; Roitberg, A.E.; Priestap, H.; Pérez, M.; et al. Molecular and functional characterization of a juvenile hormone acid methyltransferase expressed in the corpora allata of mosquitoes. Insect Biochem. Mol. Biol. 2009, 39, 31-37. [CrossRef] 
68. Minakuchi, C.; Namiki, T.; Yoshiyama, M.; Shinoda, T. RNAi-mediated knockdown of juvenile hormone acid O-methyltransferase gene causes precocious metamorphosis in the red flour beetle Tribolium castaneum. FEBS J. 2008, 275, 2919-2931. [CrossRef]

69. Niwa, R.; Niimi, T.; Honda, N.; Yoshiyama, M.; Itoyama, K.; Kataoka, H.; Shinoda, T. Juvenile hormone acid O-methyltransferase in Drosophila melanogaster. Insect Biochem. Mol. Biol. 2008, 38, 714-720. [CrossRef]

70. Sheng, Z.; Ma, L.; Li, S.; Cao, M.-X.; Jiang, R.-J. Juvenile hormone acid methyl transferase is a key regulatory enzyme for juvenile hormone synthesis in the Eri silkworm, Samia cynthica ricini. Arch. Insect Biochem. Physiol. 2008, 69, 143-154. [CrossRef]

71. Marchal, E.; Zhang, J.; Badisco, L.; Verlinden, H.; Hult, E.F.; Van Wielendaele, P.; Yagi, K.J.; Tobe, S.S.; Vanden Broeck, J. Final steps in juvenile hormone biosynthesis in the desert locust, Schistocerca gregaria. Insect Biochem. Mol. Biol. 2011, 41, 219-227. [CrossRef]

72. Kappler, C.; Goltzené, F.; Lagueux, M.; Hetru, C.; Hoffmann, J.A. Role of the follicle cells and the oocytes in ecdysone biosynthesis and esterification in vitellogenic females of Locusta migratoria. Int. J. Invertebr. Reprod. Dev. 1986, 9, 17-34. [CrossRef]

73. Lagueux, M.; Hirn, M.; Hoffmann, J.A. Ecdysone during ovarian development in Locusta migratoria. J. Insect Physiol. 1977, 23, 109-119. [CrossRef]

74. Gilbert, L.I.; Warren, J.T. A Molecular Genetic Approach to the Biosynthesis of the Insect Steroid Molting Hormone. Vitam. Horm. 2005, 73, 31-57. [CrossRef] [PubMed]

75. Niwa, R.; Matsuda, T.; Yoshiyama, T.; Namiki, T.; Mita, K.; Fujimoto, Y.; Kataoka, H. CYP306A1, a Cytochrome P450 Enzyme, Is Essential for Ecdysteroid Biosynthesis in the Prothoracic Glands ofBombyxandDrosophila. J. Biol. Chem. 2004, 279, 35942-35949. [CrossRef] [PubMed]

76. Warren, J.T.; Petryk, A.; Marqués, G.; Parvy, J.-P.; Shinoda, T.; Itoyama, K.; Kobayashi, J.; Jarcho, M.; Li, Y.; O'Connor, M.B.; et al. Phantom encodes the 25-hydroxylase of Drosophila melanogaster and Bombyx mori: A P450 enzyme critical in ecdysone biosynthesis. Insect Biochem. Mol. Biol. 2004, 34, 991-1010. [CrossRef] [PubMed]

77. Ono, H.; Rewitz, K.F.; Shinoda, T.; Itoyama, K.; Petryk, A.; Rybczynski, R.; Jarcho, M.; Warren, J.T.; Marqués, G.; Shimell, M.J.; et al. Spook and Spookier code for stage-specific components of the ecdysone biosynthetic pathway in Diptera. Dev. Biol. 2006, 298, 555-570. [CrossRef]

78. Hult, E.F.; Huang, J.; Marchal, E.; Lam, J.; Tobe, S.S. RXR/USP and EcR are critical for the regulation of reproduction and the control of JH biosynthesis in Diploptera punctata. J. Insect Physiol. 2015, 80, 48-60. [CrossRef]

79. Bellés, X.; Cassier, P.; Cerda, X.; Pascual, N.; Andre, M.; Rósso, Y.; Piulachs, M. Induction of choriogenesis by 20-hydroxyecdysone in the german cockroach. Tissue Cell 1993, 25, 195-204. [CrossRef]

80. Lange, A.B. Neural mechanisms coordinating the female reproductive system in the locust. Front Biosci 2009, 14, 4401-4415. [CrossRef]

81. Davey, K.G. The interaction of feeding and mating in the hormonal control of egg production in Rhodnius prolixus. J. Insect Physiol. 2007, 53, 208-215. [CrossRef]

82. Wang, F.; Wang, K.; Forknall, N.; Patrick, C.; Yang, T.; Parekh, R.; Bock, D.; Dickson, B. Neural circuitry linking mating and egg laying in Drosophila females. Nature 2020, 579, 101-105. [CrossRef]

83. Bergerard, J.; Seugé, J. La parthénogenèse accidentelle chez Locusta migratoria L. Bull. Biol. Fr. Belg. 1959, 93, 16-37.

84. Pardo, M.C.; López-León, M.D.; Cabrero, J.; Camacho, J.P. Cytological and developmental analysis of tychoparthenogenesis in Locusta migratoria. Heredity 1995, 75, 485-494. [CrossRef]

85. Vandesompele, J.; De Preter, K.; Pattyn, F.; Poppe, B.; Van Roy, N.; De Paepe, A.; Speleman, F. Accurate normalization of real-time quantitative RT-PCR data by geometric averaging of multiple internal control genes. Genome Biol. 2002. [CrossRef] [PubMed]

86. Billen, J. Morphology and ultrastructure of the Dufour gland in workers of social wasps (Hymenoptera, Vespidae). Arthropod Struct. Dev. 2006, 35, 77-84. [CrossRef] [PubMed]

(C) 2020 by the authors. Licensee MDPI, Basel, Switzerland. This article is an open access article distributed under the terms and conditions of the Creative Commons Attribution (CC BY) license (http://creativecommons.org/licenses/by/4.0/). 